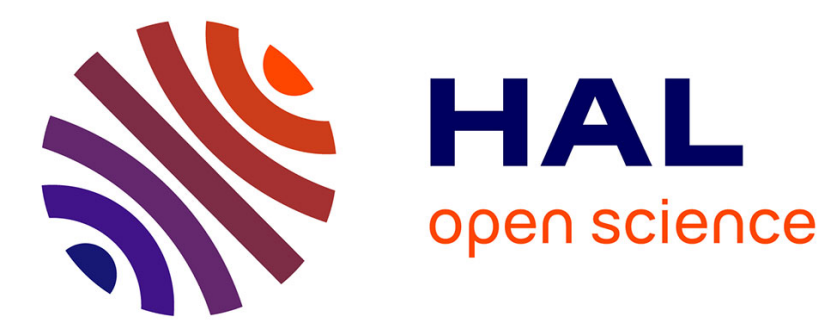

\title{
On a variational problem with lack of compactness: the topological effect of the critical points at infinity
}

\author{
Abbas Bahri, Yanyan Li, Olivier Rey
}

\section{To cite this version:}

Abbas Bahri, Yanyan Li, Olivier Rey. On a variational problem with lack of compactness: the topological effect of the critical points at infinity. Calculus of Variations and Partial Differential Equations, 1995, 3, pp.67-94. hal-00943456

\section{HAL Id: hal-00943456 https://hal.science/hal-00943456}

Submitted on 22 Dec 2016

HAL is a multi-disciplinary open access archive for the deposit and dissemination of scientific research documents, whether they are published or not. The documents may come from teaching and research institutions in France or abroad, or from public or private research centers.
L'archive ouverte pluridisciplinaire HAL, est destinée au dépôt et à la diffusion de documents scientifiques de niveau recherche, publiés ou non, émanant des établissements d'enseignement et de recherche français ou étrangers, des laboratoires publics ou privés. 


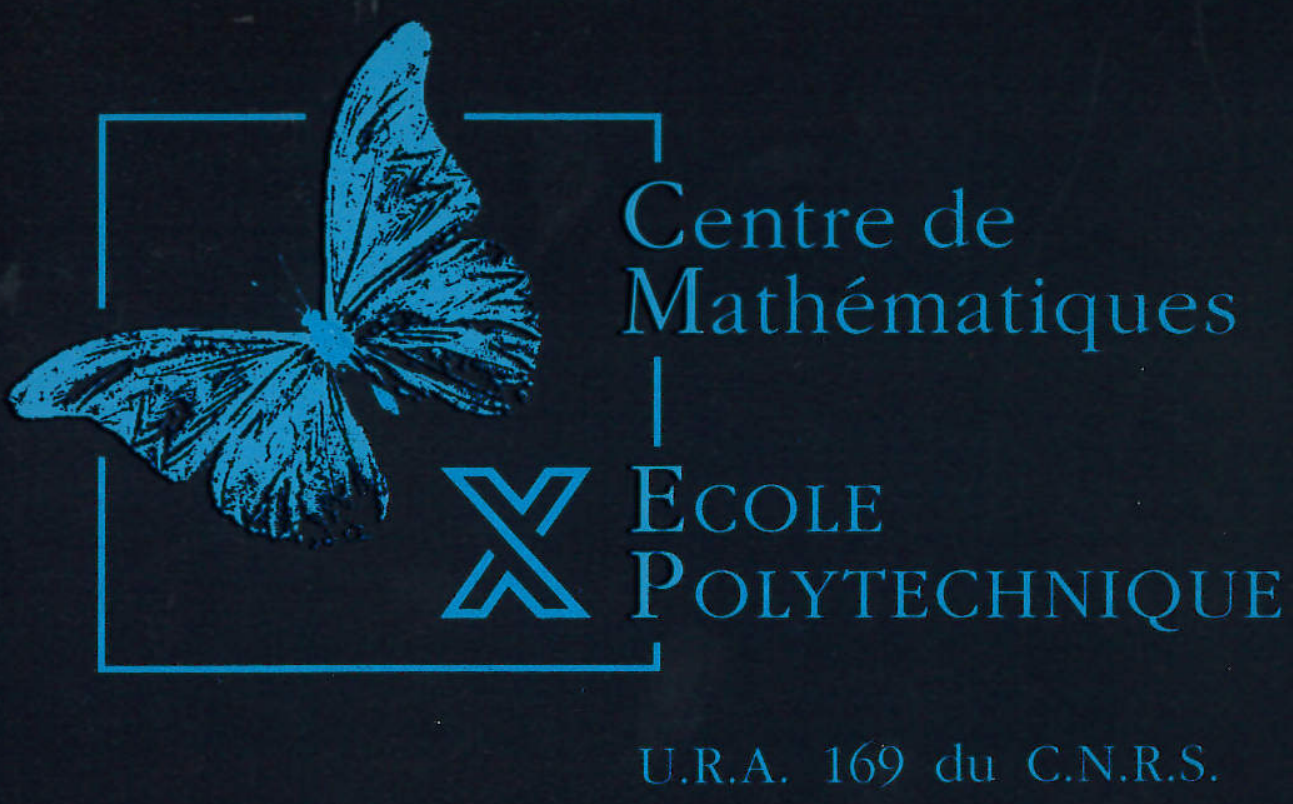

On a variational problem with lack of compactness : the topological effect of the critical points at infinity

Abbas BAHRI, Yanyan LI, Olivier REY 
RÉSumÉ. - Nous étudions les problèmes sous-critiques $\left(P_{\varepsilon}\right):-\Delta u=$ $u^{p-\varepsilon}, u>0$ sur $\Omega ; u=0$ sur $\partial \Omega$ - où $\Omega$ est un domaine borné et régulier de $\mathrm{R}^{N}, N \geq 3, p+1=\frac{2 N}{N-2}$ est l'exposant critique de Sobolev, et $\varepsilon>0$ tend vers zéro, afin de calculer la différence de toplogie induite par les points critiques à l'infini entre les ensembles de niveau de la fonctionnelle correspondant au cas limite $\left(P_{0}\right)$.

AbStraCt. - We study the subcritical problems $\left(P_{\varepsilon}\right):-\Delta u=u^{p-\varepsilon}, u>$ 0 on $\Omega ; u=0$ on $\partial \Omega, \Omega$ being a smooth and bounded domain in $\mathrm{R}^{N}, N \geq$ $3, p+1=\frac{2 N}{N-2}$ the critical Sobolev exponent and $\varepsilon>0$ going to zero - in order to compute the difference of topology that the critical points at infinity induce between the level sets of the functional corresponding to the limit case $\left(P_{0}\right)$.

Classification AMS. - $\quad 35$ J 65

Mots-CLÉs. - nonlinear elliptic equations, critical points at infinity, limiting Sobolev exponent. 


\section{Introduction}

In this paper, we come back to the study of the nonlinear elliptic problem

$$
(P)\left\{\begin{aligned}
-\Delta u & =u^{p}, u>0 & & \text { on }
\end{aligned}\right.
$$

where $\Omega$ is a smooth and bounded domain in $\mathbb{R}^{N}, N \geq 3$, and $p=\frac{N+2}{N-2}$. The interest in this equation grew up from its resemblance to the Yamabe problem in differential geometry, which consists in finding $u>0$ satisfying

$$
-\Delta u=u^{p}-\frac{N-2}{4(N-1)} R(x) u \quad \text { on } \quad M
$$

where $M$ is a Riemannian manifold of dimension $N$ without boundary and $R(x)$ is the scalar curvature (see [34] [2] [17] for example). In contrast with the subcritical case $p<\frac{N+2}{N-2}$, the variational problem corresponding to $(P)$ happens to be lacking of compactness, i.e. the functionals that we consider do not satisfy the PalaisSmale condition (P.S.). This means that there exist sequences along which they are bounded, their gradient goes to zero, and which do not converge. In the case of $(P)$ such a fact follows from the noncompactness of the embedding of $H_{0}^{1}(\Omega)$ into $L^{p+1}(\Omega)$. As well for the complete solution of Yamabe's conjecture by $\mathrm{R}$. Schoen [30] as for $(P)$ modified with a linear term $a(x) u[8]$, it is possible to make the functionals smaller than a certain level under which (P.S.) holds. Such an argument is not available for $(P)$, so that questions related to existence or nonexistence and multiplicity of solutions to this problem remained open.

The first result concerning $(P)$ was obtained by Pohozaev [24] : he proved that the problem had no solutions under the assumption that $\Omega$ is starshaped. On the other hand, Kazdan and Warner [17] observed that $(P)$ had a (radial) solution when $\Omega$ is an annulus ; hence the idea of exploiting the topology of $\Omega$ to establish the existence of a solution. This program was realized by Bahri and Coron [7], who proved that $(P)$ had a solution provided that $\Omega$ has nontrivial topology, in the sense that $H_{2 k-1}(\Omega ; \mathbf{Q}) \neq 0$ or $H_{k}(\Omega ; \mathrm{Z} / 2 \mathrm{Z}) \neq 0$ for some $k \in \mathbf{N}^{*}$. Nevertheless, Ding [12] (see also Dancer [11]) gave the example of contractible domains on which a solution still exists, showing that both topology and geometry of the domain play a role. In fact, the good condition for existence and multiplicity of solutions should involve the Green's function of the Laplacian with Dirichlet boundary conditions on $\Omega$, the importance of which in this kind of problems has already been pointed out, see e.g. [6] [3].

As we said, the problem we are interested in is delicate from a variational viewpoint because of the failure of the (P.S.) condition, more precisely because of the possible existence of critical points at infinity, that is orbits of the considered functional along which the functional is bounded, its gradient goes to zero, and which do not converge [4] [5]. If we try to prove the existence of critical points by looking at the difference of topology which occurs between the level sets of the functional, it 
becomes essential to determine the part which has to be attributed in these differences to the critical points at infinity. The strategy that we develop in this direction relies on the approximation of $(P)$ by the subcritical problems

$$
\left(P_{\varepsilon}\right)\left\{\begin{aligned}
-\Delta u & =u^{p-\varepsilon}, u>0 & & \text { on }
\end{aligned}\right.
$$

$\varepsilon>0$, for which (P.S.) holds. To the critical points at infinity which may occur in the variational formulation of $(P)$ correspond critical points in the usual sense of the functional

$$
J_{\varepsilon}(u)=\frac{1}{2} \int_{\Omega}|\nabla u|^{2}-\frac{1}{p+1-\varepsilon} \int_{\Omega}|u|^{p+1-\varepsilon}, \forall u \in H_{0}^{1}(\Omega)
$$

whose positive critical points are solutions of $\left(P_{\varepsilon}\right)$. Such solutions exist (see e.g. [21]), and as $\varepsilon$ goes to zero they may either converge to a solution of $(P)$, or blow-up at a finite number of points of $\Omega$. More precisely, if we assume that $\left(u_{\varepsilon}\right)$ is a bounded sequence in $H_{0}^{1}(\Omega)$ of solutions to $\left(P_{\varepsilon}\right)$, then (up to a subsequence) we have:

$$
u_{\varepsilon}=u_{0}+\sum_{i=1}^{k} \alpha_{i}^{\varepsilon} P \delta_{\lambda_{i}^{\varepsilon}, x_{i}^{\varepsilon}}+v^{\varepsilon}
$$

where $u_{0}$ is either a solution to $(P)$ or is identically zero, $v^{\varepsilon}$ goes to zero in $H_{0}^{1}(\Omega)$ and $k \in \mathrm{N}$. Let us describe the singular part which occurs if $k \neq 0$ :

$\alpha_{i}^{\varepsilon} \in \mathbf{R}$, and

$$
\alpha_{i}^{\varepsilon} \rightarrow \alpha=(N(N-2))^{\frac{N-2}{4}}
$$

$$
\lambda_{i}^{\varepsilon} \in \mathbf{R}_{+}^{*}, x_{i}^{\varepsilon} \in \Omega, x_{i}^{\varepsilon} \rightarrow x_{i} \in \bar{\Omega} \text { and }
$$

$$
\lambda_{i}^{\varepsilon} d\left(x_{i}^{\varepsilon}, \partial \Omega\right) \rightarrow+\infty
$$

$$
\frac{\lambda_{i}^{\varepsilon}}{\lambda_{j}^{\varepsilon}}+\frac{\lambda_{j}^{\varepsilon}}{\lambda_{i}^{\varepsilon}}+\lambda_{i}^{\varepsilon} \lambda_{j}^{\varepsilon}\left|x_{i}^{\varepsilon}-x_{j}^{\varepsilon}\right|^{2} \rightarrow+\infty, i \neq j
$$

Lastly, for $x \in \mathbf{R}^{N}$ and $\lambda>0, P \delta_{\lambda, x}$ denotes the projection onto $H_{0}^{1}(\Omega)$ of the function

$$
\begin{aligned}
\delta_{\lambda, x}: \mathbf{R}^{N} & \rightarrow \mathbf{R} \\
y & \longmapsto \lambda^{\frac{N-2}{2}}\left(1+\lambda^{2}|y-x|^{2}\right)^{-\frac{N-2}{2}}
\end{aligned}
$$


defined by

$$
\Delta P \delta_{\lambda, x}=\Delta \delta_{\lambda, x} \quad \text { on } \Omega ; P \delta_{\lambda, x}=0 \quad \text { on } \partial \Omega
$$

The functions $\alpha \delta_{\lambda, x}$ are the only solutions of the equation

$$
-\Delta u=u^{p}, u>0, \quad \text { on } \mathbf{R}^{N}
$$

(See e.g. [1] [33] [21] [10] [19]). They are also the only minimizers for the Sobolev inequality ([23] [14] [13])

$$
\begin{gathered}
S=\inf _{\nabla u \in L^{2}, u \in L^{p+1}, u \neq 0}|\nabla u|_{L^{2}\left(\mathbf{R}^{N}\right)}^{2}|u|_{L^{p+1}\left(\mathbf{R}^{N}\right)}^{-2} \\
=\inf _{u \in H_{0}^{1}(\Omega), u \neq 0}|u|_{H_{0}^{1}(\Omega)}^{2}|u|_{L^{p+1}(\Omega)}^{-2}
\end{gathered}
$$

and responsible of the failure of (P.S.) for $J_{0}$

A decomposition as in (2) is given in [22] [32]. Heuristically speaking, condition (4) means that the boundary effect is small with respect to the concentration effect, and condition (5), introduced in [7], that at first order the $P \delta_{\lambda_{i}^{\varepsilon}, x_{i}^{\epsilon}}$ 's behave independently of each other. Besides these results we have the estimates

$$
\begin{aligned}
& \left|u_{\varepsilon}\right|_{H_{0}^{1}}^{2}=\left|u_{0}\right|_{H_{0}^{1}}^{2}+k A+\circ(1) \\
& J_{\varepsilon}\left(u_{\varepsilon}\right)=J_{0}\left(u_{0}\right)=k B+\circ(1)
\end{aligned}
$$

where $A, B$ are constants which depend on $N$ only :

$$
\begin{aligned}
& A=\left|\nabla\left(\alpha \delta_{\lambda, x}\right)\right|_{L^{2}\left(\mathbf{R}^{N}\right)}^{2}=S^{N / 2} \\
& B=\frac{1}{2}\left|\nabla\left(\alpha \delta_{\lambda, x}\right)\right|_{L^{2}\left(\mathbf{R}^{N}\right)}^{2}-\frac{1}{p+1}\left|\alpha \delta_{\lambda, x}\right|_{L^{p+1}\left(\mathbf{R}^{N}\right)}^{p+1}=\frac{S^{N / 2}}{N}
\end{aligned}
$$

In fact, a recent result of $\mathrm{R}$. Schoen [31] provides us with a new and precious information: in our setting we have the alternative

$$
\text { either } k=0, \quad \text { or } \quad u_{0} \equiv 0
$$

In the following we are interested in the case $k \neq 0$ (and then $u_{0} \equiv 0$ ), i.e. in the solution to $\left(P_{\varepsilon}\right)$ which blow-up at $k$ points $x_{1}, \cdots, x_{k}$ of $\bar{\Omega}$ as $\varepsilon$ goes to zero. In a first step, we give a precise characterization of the points at which the $k$ singular solutions $u_{\varepsilon}$ of $\left(P_{\varepsilon}\right)$ blow-up, in the sense, according to $(2,3,4,5)$ :

$$
\left|\nabla u_{\varepsilon}\right|^{2} \underset{\varepsilon \rightarrow 0}{\longrightarrow} S^{N / 2} \sum_{i=1}^{k} \delta_{x_{i}} ; u_{\varepsilon}^{p+1} \underset{\varepsilon \rightarrow 0}{\longrightarrow} S^{N / 2} \sum_{i=1}^{k} \delta_{x_{i}}
$$


in the sense of measures, where $\delta_{x_{i}}$ denotes the Dirac measure at $x_{i}$. From (9) (10) we know that $J_{\varepsilon}\left(u_{\varepsilon}\right)$ converges to

$$
c_{k}=k B=k \frac{S^{N / 2}}{N}
$$

In a second step we take advantage of the description of the $k$-singular solutions that we performed to compute the difference of topology induced by these solutions between the level sets of the functional $J_{\varepsilon}$ across the level $c_{k}$, namely between

$$
J_{\varepsilon}^{c_{k}+\eta}=\left\{u \in H_{0}^{1}(\Omega) / J_{\varepsilon}(u) \leq c_{k}+\eta\right\}
$$

and

$0<\eta<\frac{S^{N / 2}}{N}$

$$
J_{\varepsilon}^{c_{k}-\eta}=\left\{u \in H_{0}^{1}(\Omega) / J_{\varepsilon}(u) \leq c_{k}-\eta\right\}
$$

\section{Results}

Before stating the results, we need some notations. We denote by $G$ the Green's function of the Laplacian with Dirichlet boundary condition on $\Omega$, and by $H$ its regular part, i.e.

$$
G(x, y)=\frac{1}{|x-y|^{N-2}}-H(x, y) \quad \text { for } \quad(x, y) \in \Omega \times \Omega
$$

and

$$
\Delta_{x} H=0 \quad \text { on } \Omega \times \Omega, G=0 \text { on } \partial(\Omega \times \Omega)
$$

For $k \in \mathbf{N}^{*}$ and $\mathbf{x}=\left(x_{1}, \cdots, x_{k}\right) \in \Omega^{k}$, we denote by $M(\mathbf{x})=\left(m_{i j}\right)_{1 \leq i, j \leq k}$ the matrix defined by

$$
m_{i i}=H\left(x_{i}, x_{i}\right) ; m_{i j}=-G\left(x_{i}, x_{j}\right), i \neq j
$$

by $\rho(\mathbf{x})$ its least eigenvalue $\left(\rho(\mathbf{x})=-\infty\right.$ if $x_{i}=x_{j}$ for some $\left.i \neq j\right)$, and by $r(\mathbf{x})$ the eigenvector corresponding to $\rho(\mathrm{x})$ whose norm is 1 and whose components all strictly positive (see Appendix A for the proof that $\rho$ is simple and that one can choose $r$ so that all its components are stricly positive). We also define the function

$$
\begin{aligned}
F_{\mathbf{X}} \quad:\left(\mathbf{R}_{+}^{*}\right)^{k} & \rightarrow \mathbf{R} \\
\Lambda=\left(\Lambda_{1}, \cdots \Lambda_{k}\right) & \mapsto \frac{1}{2} \Lambda M(\mathbf{x})^{t} \Lambda-\log \Lambda_{1} \ldots \Lambda_{k}
\end{aligned}
$$

If $\rho(\mathbf{x})>0, F_{\mathbf{x}}$ is strictly convex on $\left(\mathbf{R}_{+}^{*}\right)^{k}$, infinite on the boundary ; so $F_{\mathbf{x}}$ has in $\left(\mathbf{R}_{+}^{*}\right)^{k}$ a unique critical point $\Lambda(\mathrm{x})$, which is a minimum. On the subset of $\Omega^{k}$

$$
\rho^{+}=\left\{\mathrm{x} \in \Omega^{k} / \rho(\mathrm{x})>0\right\}
$$


we define the function

$$
\tilde{F}(\mathrm{x})=F_{\mathrm{x}}(\Lambda(\mathrm{x}))=\frac{k}{2}-\log \Lambda_{1}(\mathrm{x}) \ldots \Lambda_{k}(\mathrm{x})
$$

whose differential is given by

$$
\tilde{F}^{\prime}(\mathrm{x})=\frac{1}{2} \Lambda(\mathrm{x}) M^{\prime}(\mathrm{x})^{t} \Lambda(\mathrm{x})=-\sum_{i=1}^{k} \frac{\Lambda_{i}^{\prime}(\mathrm{x})}{\Lambda_{i}(\mathrm{x})}
$$

Now we are able to state the following results

Theorem 1. - Assume that $N \geq 4$. Let $\left(u_{\varepsilon}\right)$ be a sequence of solution to $\left(P_{\varepsilon}\right)$ which blow-up at $k$ points $x_{1}, \cdots, x_{k} \in \bar{\Omega}$ as $\epsilon \rightarrow 0$. Then

(a) $\mathrm{x}=\left(x_{1}, \cdots, x_{k}\right) \in \Omega_{d_{0}}^{k}$, with $d_{0}=d_{0}(\Omega)>0$ and $\Omega_{d_{0}}=\left\{x \in \Omega / d(x, \partial \Omega)>d_{0}\right\}$

(b) $\rho(\mathbf{x}) \geq 0$

(c) either $\rho(\mathrm{x})>0$ and $\tilde{F}^{\prime}(\mathrm{x})=0$, or $\rho(\mathrm{x})=0$ and $\rho^{\prime}(\mathrm{x})=0$

The index of these solutions as critical points of $J_{\varepsilon}$ is at least $\ell+k$ (and at most $(N+1) k)$, where $\ell$ is the index of $\mathbf{x}$ as a critical point of $\tilde{F}$ (or $\rho$ ). If $\mathbf{x}$ is a nondegenerate critical point of $\tilde{F}$, the index of $u_{\varepsilon}$ is exactly $\ell+k$.

Conversely, if $\mathrm{x} \in \rho^{+}$is a nondegenerate critical point of $\tilde{F}$, there exists for $\varepsilon$ small enough a sequence of solutions to $\left(P_{\varepsilon}\right)$ which blow-up at $x_{1}, \cdots, x_{k}$ as $\varepsilon \rightarrow 0$.

Under the assumption that 0 is a regular value for $\rho,(b)$ and $(c)$ may be replaced by

(b') $\rho(\mathbf{x}) \geq \rho_{0}(\Omega)>0$

(c') $\tilde{F}^{\prime}(\mathbf{x})=0$

Moreover, if $\rho(\mathbf{x})>0$, we have the estimates

$$
\begin{gathered}
\frac{1}{\left(\lambda_{i}^{\varepsilon}\right)^{\frac{N-2}{2}}} \sim \gamma \Lambda_{i}(\mathrm{x}) \varepsilon^{1 / 2} \\
J_{\varepsilon}\left(u_{\varepsilon}\right)=c_{k}+k \gamma_{1} \varepsilon|\log \varepsilon|+k \gamma_{2} \varepsilon+2 \gamma_{1} \varepsilon \tilde{F}(\mathrm{x})+o(\varepsilon)
\end{gathered}
$$

$\gamma>0, \gamma_{1}>0, \gamma_{2}$ constants which depend on $N$ only

Remarks : 1 - In the case $k=1, M(x)=H(x, x)>0$ everywhere, $\Lambda(x)=$ $H(x, x)^{-1 / 2}$, and $\tilde{F}(x)=\frac{1}{2}+\frac{1}{2} \log H(x, x)$. Solutions to $\left(P_{\varepsilon}\right)$ which blow-up at one point as $\varepsilon \rightarrow 0$ always exist - see e.g. [26] - and we find again that they concentrate at a critical point of $\varphi(x)=H(x, x)$ - see [9] [16] [29].

2 - In the case $k=2, M(\mathrm{x})=\left(\begin{array}{cc}H\left(x_{1}, x_{1}\right) & -G\left(x_{1}, x_{2}\right) \\ -G\left(x_{1}, x_{2}\right) & H\left(x_{2}, x_{2}\right)\end{array}\right)$,

$$
\Lambda_{i}^{2}(\mathrm{x})=\frac{H\left(x_{j}, x_{j}\right)^{1 / 2}}{H\left(x_{i}, x_{i}\right)^{1 / 2}} \frac{1}{\phi(\mathrm{x})}, i \neq j, \quad \text { and } \quad \tilde{F}(\mathrm{x})=1+\log \phi(\mathrm{x}) \text {, }
$$


with

$$
\phi(\mathbf{x})=H\left(x_{1}, x_{1}\right)^{1 / 2} H\left(x_{2}, x_{2}\right)^{1 / 2}-G\left(x_{1}, x_{2}\right)
$$

As

$$
\rho(\mathbf{x})=\frac{1}{2}\left[H\left(x_{1}, x_{1}\right)+H\left(x_{2}, x_{2}\right)-\left(\left(H\left(x_{1}, x_{1}\right)-H\left(x_{2}, x_{2}\right)\right)^{2}+4 G\left(x_{1}, x_{2}\right)^{2}\right)^{1 / 2}\right]
$$

$\rho(\mathbf{x})>0$ is equivalent to $\phi(\mathbf{x})>0$, and (b) (c) may be replaced in Theorem 1 , for $k=2$, by

$$
\phi(\mathbf{x}) \geq 0 ; \phi^{\prime}(\mathbf{x})=0
$$

(since for $\rho=0, \rho^{\prime}=0$ is equivalent also to $\phi^{\prime}=0$, as a simple computation shows). This result was presented in [29].

3 - For $k$ large enough, there are no solutions to $\left(P_{\varepsilon}\right)$ blowing-up at $k$ points as $\varepsilon \rightarrow 0$. Indeed, conditions (a) and (b) cannot be satisfied simultaneously ; $H(x, x)$ is bounded on $\Omega_{d_{0}}$ whereas $G(x, y)$ goes to $-\infty$ if $x, y \in \Omega_{d_{0}}$ get close from each other ; then, for $k$ large enough, $\rho(\mathrm{x})<0$ on $\Omega_{d_{0}}^{k}$.

Once we know the results of Theorem 1, we are able to prove :

Theorem 2. - Assume that $N \geq 4$, and that 0 is a regular value of $\rho$.

The contribution to the relative homology

$$
H_{*}\left(J_{\varepsilon}^{c_{k}+\eta}, J_{\varepsilon}^{c_{k}-\eta}\right)
$$

of the solutions of $\left(P_{\varepsilon}\right)$ which blow-up at $k$ points as $\varepsilon$ goes to zero, $0<\eta<\frac{S^{N / 2}}{N}$, is equal for $\varepsilon$ small enough to

$$
H_{*}\left(\left(\Omega^{k}, \rho^{-}\right) \times\left(D^{k}, S^{k-1}\right)\right)
$$

with

$$
\rho^{-}=\left\{\mathrm{x} \in \Omega^{k} / \rho(\mathrm{x}) \leq 0\right\}
$$

This result points out the importance of the behavior of the least eigenvalue $\rho(\mathrm{x})$ of the matrix $M(\mathrm{x})$ on $\Omega^{k}$, which already appeared in [6] [3] (see also [27] $[28]$ ). It shows in particular, since the functionals $J_{\varepsilon}$ satisfy (P.S.) and the difference of topology between the level sets may only come from critical points, that if $\left(\Omega^{k}, \rho^{-}\right)$is nontrivial, $\left(P_{\varepsilon}\right)$ does have, as $\varepsilon$ goes to zero, solutions which blow-up at $k$ points. This gives a meaning to Theorem 1 without any further assumption of nondegeneracy etc. (Note that for $k=1, H_{N}\left(\Omega, \rho^{-}\right)=H_{N}(\Omega) \neq 0$ ). The stability of the result that we obtain with $\varepsilon$, will allow us to transfer it to the functional $J_{0}$. Then, Theorem 2 should make us able, through the study of the Green's function, to answer to questions related to existence and multiplicity of solutions to $(P)$, this last question raised by R.Bott. 


\section{The general framework}

The framework is similar to the one considered in [29]. For $a>0$, we define the subset of $H_{0}^{1}(\Omega)$

$$
\begin{aligned}
& F_{a}=\left\{\alpha \sum_{i=1}^{k} P \delta_{\lambda_{i}, x_{i}} / \lambda_{i} d\left(x_{i}, \partial \Omega\right)>\frac{1}{a}, \forall i\right. \\
& \left.\frac{\lambda_{i}}{\lambda_{j}}+\frac{\lambda_{j}}{\lambda_{i}}+\lambda_{i} \lambda_{j}\left|x_{i}-x_{j}\right|^{2}>\frac{1}{a}, \forall i, j, i \neq j\right\}
\end{aligned}
$$

with $\alpha=(N(N-2))^{\frac{N-2}{4}}$ and $P \delta_{\lambda, x} \in H_{0}^{1}(\Omega)$ as stated in the Introduction, i.e.

$$
P \delta_{\lambda, x}=\delta_{\lambda, x}-\varphi_{\lambda, x}
$$

$\delta_{\lambda, x}$ being defined by (6) and $\varphi_{\lambda, x}$ such that

$$
\Delta \varphi_{\lambda, x}=0 \quad \text { on } \quad \Omega ; \varphi_{\lambda, x}=\delta_{\lambda, x} \quad \text { on } \quad \partial \Omega
$$

The maximum principle yields

$$
\varphi_{\lambda, x}(y)=\frac{1}{\lambda^{\frac{N-2}{2}}} H(x, y)+0\left(\frac{1}{\lambda^{\frac{N+2}{2}}}\right), d=d(x, \partial \Omega)
$$

If $\left(u_{\varepsilon}\right)$ is a sequence of solutions to $\left(P_{\varepsilon}\right)$ which blow-up at $k$ points of $\bar{\Omega}$ as $\varepsilon$ goes to zero, it follows from what was said in the Introduction that for any $a>0$ fixed, the distance between $u_{\varepsilon}$ and $F_{a}$ goes to zero with $\varepsilon$. Moreover, it is proved in [7] that if $u \in H_{0}^{1}(\Omega)$ satisfies $\operatorname{dist}_{H_{0}^{1}(\Omega)}\left(u, F_{a}\right)<a$ with $a$ small enough, the problem :

$$
\text { Minimize }\left|u-\sum_{i=1}^{k} \alpha_{i} P \delta_{\lambda_{i}, x_{i}}\right|_{H_{0}^{1}} \quad \text { with respect to the } \alpha_{i}, \lambda_{i}, x_{i}^{\prime} s
$$

has a unique solution in the open set:

$$
\begin{gathered}
\left|\alpha_{i}-\alpha\right|<4 a, \lambda_{i} d\left(x_{i}, \partial \Omega\right)>\frac{1}{4 a}, \forall i \\
\frac{\lambda_{i}}{\lambda_{j}}+\frac{\lambda_{i}}{\lambda_{j}}+\lambda_{i} \lambda_{j}\left|x_{i}-x_{j}\right|^{2}>\frac{1}{4 a}, \forall i, j, i \neq j
\end{gathered}
$$

Therefore, there exist a diffeomorphism between a neighborhood of the possible $k$-singular solutions of $\left(P_{\varepsilon}\right)$ we are intersted in and the open set

$$
\begin{gathered}
M=\left\{m=(\alpha, \lambda, \mathbf{x}, v) \in \mathbf{R}^{k} \times\left(\mathbf{R}_{+}^{*}\right)^{k} \times \Omega^{k} \times H_{0}^{1}(\Omega) /\right. \\
\left|\alpha_{i}-\alpha\right|<\nu_{0}, \lambda_{i} d\left(x_{i}, \partial \Omega\right)>\frac{1}{\nu_{0}}, \forall i ; \\
\left.\frac{\lambda_{i}}{\lambda_{j}}+\frac{\lambda_{i}}{\lambda_{i}}+\lambda_{i} \lambda_{j}\left|x_{i}-x_{j}\right|^{2}>\frac{1}{\nu_{0}}, \forall i, j, i \neq j ; v \in E_{\lambda, \mathbf{x}},|v|_{H_{0}^{T}}<\nu_{0}\right\}
\end{gathered}
$$


with $\nu_{0}>0$ some suitable constant and

$$
E_{\lambda, \mathbf{x}}=\left\{v \in H_{0}^{1}(\Omega) /\langle v, P \delta i\rangle_{H_{0}^{1}}=\left\langle v, \frac{\partial P \delta_{i}}{\partial \lambda_{i}}\right\rangle_{H_{0}^{1}}=\left\langle v, \frac{\partial P \delta_{i}}{\partial x_{i}}\right\rangle_{H_{0}^{1}}=0, \forall i\right\}
$$

(we write $P \delta_{i}=P \delta_{\lambda_{i}, x_{i}}$ )

$u_{\varepsilon}$ solution to $\left(P_{\varepsilon}\right)$ and satisfying the assumptions of Theorem 1 may be written, for $\varepsilon$ small enough, as

$$
u_{\varepsilon}=\sum_{i=1}^{k} \alpha_{i}^{\varepsilon} P \delta_{\lambda_{i}^{\varepsilon}, x_{i}^{\varepsilon}}+v^{\varepsilon}
$$

with $m^{\varepsilon}=\left(\alpha^{\varepsilon}, \lambda^{\varepsilon}, \mathbf{x}^{\varepsilon}, v^{\varepsilon}\right) \in M$, and the following properties

$$
\alpha_{i}^{\varepsilon} \rightarrow \alpha, \lambda_{i}^{\varepsilon} d\left(x_{i}^{\varepsilon}, \partial \Omega\right) \rightarrow+\infty, \frac{\lambda_{i}^{\varepsilon}}{\lambda_{j}^{\varepsilon}}+\frac{\lambda_{j}^{\varepsilon}}{\lambda_{i}^{\varepsilon}}+\lambda_{i}^{\varepsilon} \lambda_{j}^{\varepsilon}\left|x_{i}^{\varepsilon}-x^{\varepsilon}\right|^{2} \rightarrow+\infty, v^{\varepsilon} \rightarrow 0
$$

One can even get more precisions :

1) It is proved in [29], using an argument of Z.C. Han [16], that there exists $d_{0}=d_{0}(\Omega)>0$ such that for $\varepsilon$ small enough

$$
d\left(x_{i}^{\varepsilon}, \partial \Omega\right)>d_{0}, \forall i
$$

2) On the other hand, it follows from recent results of R. Schoen [31] that there exist $d_{0}^{\prime}(\Omega)>0$ and $c_{0}(\Omega)>0$ such that for $\varepsilon$ small enough

$$
\left|x_{i}^{\varepsilon}-x_{j}^{\varepsilon}\right|>d_{0}^{\prime}, \frac{\lambda_{i}^{\varepsilon}}{\lambda_{j}^{\varepsilon}}<c_{0} \forall i, j, i \neq j
$$

3) Lastly, using (28), multiplying the equation $-\Delta u_{\varepsilon}=u_{\varepsilon}^{p-\varepsilon}$ by $P \delta_{\lambda_{i}^{\varepsilon}, x_{i}^{\varepsilon}}$ and integrating on $\Omega$, we obtain

$$
C_{N}+0(1)=\frac{C_{N}}{\left(\lambda_{i}^{\varepsilon}\right)^{\frac{N-2}{2} \varepsilon}}+0(1)
$$

with $C_{N}=\alpha \int_{\mathbf{R}^{N}}\left|\nabla \delta_{\lambda, x}\right|^{2}=\alpha^{p} \int_{\mathbf{R}^{N}} \delta_{,, x}^{p+1}$. This implies

$$
\varepsilon \log \lambda_{i}^{\varepsilon} \rightarrow 0 \text { as } \varepsilon \rightarrow 0, \quad \forall i
$$

Finally, we see that there exist a diffeomorphism between a neighborhood of the possible solutions to $\left(P_{\varepsilon}\right)$ satisfying the assumptions of Theorem 1 and the open set

$$
\begin{array}{r}
M_{\varepsilon}=\left\{m=(\alpha, \lambda, \mathbf{x}, v) \in \mathbf{R}^{k} \times\left(\mathbf{R}_{+}^{*}\right)^{k} \times \Omega_{d_{0}}^{k} \times H_{0}^{1}(\Omega) /\right. \\
\left|\alpha_{i}-\alpha\right|<\nu_{0}, \lambda_{i}>\frac{1}{\nu_{0}}, \varepsilon \log \lambda_{i}<\nu_{0}, \quad \forall i ; \\
\left.\frac{\lambda_{i}}{\lambda_{j}}<c_{0},\left|x_{i}-x_{j}\right|>d_{0}^{\prime}, \forall i, j, i \neq j ; v \in E_{\lambda, \mathbf{x}},|v|_{H_{0}^{1}}<\nu_{0}\right\}
\end{array}
$$


where $\nu_{0}, c_{0}, d_{0}, d_{0}^{\prime}$ are some suitable strictly positive constants.

Let us define the functional

$$
\begin{array}{ll}
K_{\varepsilon}: M_{\varepsilon} & \rightarrow \mathbb{R} \\
m=(\alpha, \lambda, \mathbf{x}, v) & \mapsto J_{\varepsilon}\left(\sum_{i=1}^{k} \alpha_{i} P \delta_{\lambda_{i}, x_{i}}+v\right)
\end{array}
$$

It follows from the previous material that we have

PROPOSITION 1.

$m=(\alpha, \lambda, \mathbf{x}, v) \in M_{\varepsilon}$ is a critical point of $K_{\varepsilon}$ if and only if $u=\sum_{i=1}^{k} \alpha_{i} P \delta_{\lambda_{i}, x_{i}}+v$ is a critical point of $J_{\varepsilon}$, i.e. if and only if there exists $(A, B, C) \in \mathbb{R}^{k} \times \mathbf{R}^{k} \times\left(\mathbf{R}^{N}\right)^{k}$ such that the following holds :

(E)

$$
\begin{aligned}
& \left(E_{\alpha_{i}}\right) \frac{\partial K_{\varepsilon}}{\alpha_{i}}=0, \forall i \\
& \left(E_{\lambda_{i}}\right) \frac{\partial K_{\varepsilon}}{\partial \lambda_{i}}=B_{i}\left(\frac{\partial^{2} P \delta_{i}}{\partial \lambda_{i}^{2}}, v\right\rangle_{H_{0}^{1}}+C_{i} \cdot\left\langle\frac{\partial^{2} P \delta_{i}}{\partial x_{i} \partial \lambda_{i}}, v\right\rangle_{H_{0}^{1}}, \forall i \\
& \left(E_{x_{i}}\right) \frac{\partial K_{\varepsilon}}{\partial x_{i}}=B_{i}\left\langle\frac{\partial^{2} P \delta_{i}}{\partial \lambda_{i} \partial x_{i}}, v\right\rangle_{H_{0}^{1}}+C_{i} \cdot\left\langle\frac{\partial^{2} P \delta_{i}}{\partial x_{i}^{2}}, v\right\rangle_{H_{0}^{1}}, \forall i \\
& \left(E_{v}\right) \frac{\partial k_{\varepsilon}}{\partial v}=\sum_{i=1}^{k}\left(A_{i} P \delta_{i}+B_{i} \frac{\partial P \delta_{i}}{\partial \lambda_{i}}+C_{i} \cdot \frac{\partial P \delta_{i}}{\partial x_{i}}\right)
\end{aligned}
$$

\section{Proof of Theorem 1}

The results of Theorem 1 will be obtained through a careful analysis of $(\mathrm{E})$ on $M_{\varepsilon}$. As usual in this type of problems, we first deal with the $v$-part of $u$, in order to show that it is negligible with respect to the concentration phenomenon. The study of $\left(E_{v}\right)$ yields :

\section{PROPOSITION 2.}

There exists a smooth map which to any $(\varepsilon, \alpha, \lambda, \mathrm{x})$ such that $(\alpha, \lambda, \mathrm{x}, 0) \in M_{\varepsilon}$ associates $\bar{v} \in E_{\lambda, \mathbf{x}},|\bar{v}|_{H_{0}^{1}}<\nu_{0}$, such that $\left(E_{v}\right)$ is satisfied for some $(A, B, C) \in$ $\mathbf{R}^{k} \times \mathbf{R}^{k} \times\left(\mathbf{R}^{N}\right)^{k}$. Such a $\bar{v}$ is unique, minimizes $K_{\varepsilon}(\alpha, \lambda, \mathbf{x}, v)$ with respect to $v$ in $\left\{v \in E_{\lambda, \mathbf{x}} /|v|_{H_{0}^{1}}<\nu_{0}\right\}$, and we have the estimate

$$
|\bar{v}|_{H_{0}^{1}}=0\left[\varepsilon+\left(\frac{1}{\lambda^{N-2}} \quad \text { if } \quad N<6 ; \frac{\log \lambda}{\lambda^{4}} \quad \text { if } \quad N=6 ; \frac{1}{\lambda^{\frac{N+2}{2}}} \quad \text { if } \quad N>6\right)\right]
$$

(for sake of simplicity, the $\lambda_{i}$ 's being of the some order, we denote by $f(\lambda)$ any quantity as $f\left(\lambda_{i}\right)$ or $\left.\sum_{i=1}^{k} f\left(\lambda_{i}\right)\right)$

The proof of such a result may be found, up to minor modifications, in [3] [25] $[29]$. 
Once $\bar{v}$ is defined by Proposition 2, we estimate the corresponding numbers $A, B, C$ by taking the scalar product in $H_{0}^{1}(\Omega)$ of $\left(E_{v}\right)$ with respectively $P \delta_{i}, \frac{\partial P \delta_{i}}{\partial \lambda_{i}}, \frac{\partial P \delta_{i}}{\partial x_{i}}$. Thus we get a quasi-diagonal system whose coefficients are given by

$$
\begin{gathered}
\int_{\Omega} \nabla P \delta_{i} \nabla P \delta_{j}=N(N-2) \Gamma_{1} \delta_{i j}+0\left(\frac{1}{\lambda^{N-2}}\right) ; \int_{\Omega} \nabla P \delta_{i} \nabla \frac{\partial P \delta_{j}}{\partial \lambda_{j}}=0\left(\frac{1}{\lambda^{N+1}}\right) \\
\int_{\Omega} \nabla P \delta_{i} \nabla \frac{\partial P \delta_{j}}{\partial x_{j}}=0\left(\frac{1}{\lambda^{N-2}}\right) ; \int_{\Omega} \nabla \frac{\partial P \delta_{i}}{\partial \lambda_{i}} \nabla \frac{\partial P \delta_{j}}{\partial \lambda_{j}}=N(N+2) \frac{\Gamma_{2}}{\lambda_{i}^{2}} \delta_{i j}+0\left(\frac{1}{\lambda^{N}}\right) \\
\int_{\Omega} \nabla \frac{\partial P \delta_{i}}{\partial \lambda_{i}} \nabla \frac{\partial P \delta_{j}}{\partial x_{j}}=0\left(\frac{1}{\lambda^{N-1}}\right) ; \int_{\Omega} \nabla \frac{\partial P \delta_{i}}{\partial x i_{i}} \nabla \frac{\partial P \delta_{j}}{\partial x_{j}}=N(N+2) \Gamma_{3} \lambda_{i}^{2} \delta_{i j}+0\left(\frac{1}{\lambda^{N-2}}\right)
\end{gathered}
$$

with $\delta_{i j}$ the Kronecker symbol and

$$
\Gamma_{1}=\int_{\mathbf{R}^{N}} \frac{d x}{\left(1+|x|^{2}\right)^{N}}, \Gamma_{2}=\frac{(N-2)^{2}}{4} \int_{\mathbf{R}^{N}} \frac{\left(1-|x|^{2}\right)^{2}}{\left(1+|x|^{2}\right)^{N+2}} d x, \Gamma_{3}=(N-2)^{2} \int_{\mathbf{R}^{N}} \frac{|x|^{2} d x}{\left(1+|x|^{2}\right)^{N+2}}
$$

The other hand side is given by

$$
\left\langle\frac{\partial K_{\varepsilon}}{\partial v}, P \delta_{i}\right\rangle_{H_{0}^{1}}=\frac{\partial K_{\varepsilon}}{\partial \alpha_{i}} ;\left\langle\frac{\partial K_{\varepsilon}}{\partial v}, \frac{\partial P \delta_{i}}{\partial \lambda_{i}}\right\rangle_{H_{0}^{1}}=\frac{1}{\alpha_{i}} \frac{\partial K_{\varepsilon}}{\partial \lambda_{i}} ;\left\langle\frac{\partial K_{\varepsilon}}{\partial v}, \frac{\partial P \delta_{i}}{\partial x_{i}}\right\rangle_{H_{0}^{1}}=\frac{1}{\alpha_{i}} \frac{\partial K_{\varepsilon}}{\partial x_{i}}
$$

Explicit computations, using (6) (24) (26) (31), and whose details may be found in [3] [25], yield

$$
\frac{\partial K_{\varepsilon}}{\partial \alpha_{i}}=-4 N \Gamma_{1} \beta_{i}+V_{\alpha_{i}}(\varepsilon, \alpha, \lambda, \mathbf{x})
$$

with

$$
\beta=\left(\beta_{1}, \cdots, \beta_{k}\right)=\left(\alpha-\alpha_{1}, \cdots, \alpha-\alpha_{k}\right)
$$

and $V_{\alpha_{i}}$ a smooth function which satisfies

$$
V_{\alpha_{1}}=0\left[\beta_{i}^{2}+\frac{1}{\lambda^{N-2}}+\varepsilon \log \lambda\right]
$$

In the same way we get

$$
\frac{\partial K_{\varepsilon}}{\partial \lambda_{i}}=\Gamma_{4} \frac{\varepsilon}{\lambda_{i}}-\Gamma_{5}\left(\frac{H\left(x_{i}, x_{i}\right)}{\lambda_{i}^{N-1}}-\sum_{j \neq i} \frac{G\left(x_{i}, x_{j}\right)}{\lambda_{i}^{N / 2} \lambda_{j}^{\frac{N-2}{2}}}\right)+V_{\lambda_{i}}(\varepsilon, \alpha, \lambda, \mathbf{x})
$$

where $V_{\lambda_{i}}$ is a smooth function verifying

$$
V_{\lambda_{1}}=0\left[\frac{1}{\lambda}\left(\frac{\log \lambda}{\lambda^{N}}+\varepsilon^{2} \log \lambda+|\beta|\left(\varepsilon+\frac{1}{\lambda^{N-2}}\right)\right)\right]
$$


and

$$
\begin{aligned}
& \Gamma_{4}=-\frac{(N-2)^{2}}{4}(N(N-2))^{N / 2} \int_{\mathrm{R}^{N}} \frac{\left(1-|x|^{2}\right) \log \left(1+|x|^{2}\right)}{\left(1+|x|^{2}\right)^{N-1}} d x>0 \\
& \Gamma_{5}=-\frac{(N+2)}{2}(N(N-2))^{N / 2} \int_{\mathbf{R}^{N}} \frac{\left(1-|x|^{2}\right)}{\left(1+|x|^{2}\right)^{\frac{N-1}{2}}} d x>0
\end{aligned}
$$

Lastly, we have

$$
\frac{\partial K_{\varepsilon}}{\partial x_{i}}=\Gamma_{6}\left(\frac{1}{\lambda_{i}^{N-2}} \frac{\partial H}{\partial a}\left(x_{i}, x_{i}\right)-\sum_{j \neq i} \frac{1}{\lambda_{i}^{\frac{N-2}{2}} \lambda_{j}^{\frac{N-2}{2}}} \frac{\partial G}{\partial a}\left(x_{i}, x_{j}\right)\right)+V_{x_{i}}(\varepsilon, \alpha, \lambda, \mathbf{x})
$$

where $\frac{\partial}{\partial a}$ and $\frac{\partial}{\partial b}$ denote the derivatives with respect to the first variable and the second variable of the functions $(a, b) \rightarrow H(a, b)$ and $(a, b) \rightarrow G(a, b) ; \Gamma_{6}=\frac{2 \Gamma_{5}}{N-2}$, and $V_{x_{i}}$ is a smooth function such that

$$
V_{x_{i}}=0\left[\varepsilon^{2} \lambda+\left(\frac{1}{\lambda^{3}} \quad \text { if } \quad N=4 ; \frac{\log \lambda}{\lambda^{N}} \quad \text { if } \quad N \geq 5\right)+\frac{|\beta|}{\lambda^{N-2}}\right]
$$

Noticing that these estimates imply

$$
\frac{\partial K_{\varepsilon}}{\partial \alpha_{i}}=0\left(|\beta|+\frac{1}{\lambda^{N-2}}+\varepsilon \log \lambda+\varepsilon^{2}\right) ; \frac{\partial K_{\varepsilon}}{\partial \lambda_{i}}=0\left(\frac{1}{\lambda^{N-2}}+\frac{\varepsilon}{\lambda}\right) ; \frac{\partial K_{\varepsilon}}{\partial x_{i}}=0\left(\frac{1}{\lambda^{N-2}}+\varepsilon^{2} \lambda\right)
$$

the solution of the system in $A, B, C$ shows that

$$
\left\{\begin{array}{l}
A=0\left(|\beta|+\frac{1}{\lambda^{N-2}}+\varepsilon \log \lambda+\varepsilon^{2}\right) \\
B=0\left(\frac{1}{\lambda^{N-3}}+\varepsilon^{2} \lambda\right) \\
C=0\left(\frac{1}{\lambda^{N}}+\frac{\varepsilon^{2}}{\lambda}\right)
\end{array}\right.
$$

This makes us able to evaluate the right hand side in the equations $\left(E_{\lambda_{i}}\right)$ and $\left(E_{x_{i}}\right)$, namely

$$
\begin{aligned}
& B_{i}\left\langle\frac{\partial^{2} P \delta_{i}}{\partial \lambda_{i}^{2}}, \bar{v}\right\rangle_{H_{0}^{1}}+C_{i} \cdot\left\langle\frac{\partial^{2} P \delta_{i}}{\partial x_{i} \partial \lambda_{i}}, \bar{v}\right\rangle_{H_{0}^{1}} \\
& \quad=0\left(\left(\frac{\left|B_{i}\right|}{\lambda_{i}^{2}}+\left|C_{i}\right|\right)|\bar{v}|_{H_{0}^{1}}\right) \\
& \quad=0\left(\left(\frac{1}{\lambda^{N-1}}+\frac{\varepsilon^{2}}{\lambda}\right)|\bar{v}|_{H_{0}^{1}}\right)
\end{aligned}
$$

and

$$
\begin{array}{r}
B_{i}\left\langle\frac{\partial^{2} P \delta_{i}}{\partial \lambda_{i} \partial x_{i}}, \bar{v}\right\rangle_{H_{0}^{1}}+C_{i} \cdot\left\langle\frac{\partial^{2} P \delta_{i}}{\partial x_{i}^{2}}, \bar{v}\right\rangle_{H_{0}^{1}} \\
=0\left(\left(\frac{1}{\lambda^{N-3}}+\lambda \varepsilon\right)|\ddot{v}|_{H_{0}^{\prime}}\right)
\end{array}
$$


since

$$
\left|\frac{\partial^{2} P \delta_{i}}{\partial \lambda_{i}^{2}}\right|_{H_{0}^{1}}=0\left(\frac{1}{\lambda_{i}^{2}}\right),\left|\frac{\partial^{2} P \delta_{i}}{\partial \lambda_{i} \partial x_{i}}\right|_{H_{0}^{1}}=0(1),\left|\frac{\partial^{2} P \delta_{i}}{\partial x_{i}^{2}}\right|_{H_{0}^{1}}=0\left(\lambda_{i}^{2}\right)
$$

Let us now prove the results of Theorem 1 . We consider a sequence $\left(u_{\varepsilon}\right)$ of solutions to $\left(P_{\varepsilon}\right)$, satisfying the assumptions of the theorem. $u_{\varepsilon}$ reads as (28) with $m^{\varepsilon}=$ $\left(\alpha^{\varepsilon}, \lambda^{\varepsilon}, \mathbf{x}^{\varepsilon}, v^{\varepsilon}\right) \in M_{\varepsilon}$, and it follows from Proposition 2 that $v^{\varepsilon}=\bar{v}\left(\varepsilon, \alpha^{\varepsilon}, \lambda^{\varepsilon}, \mathbf{x}^{\varepsilon}\right)$. Then, $\left(E_{\alpha_{i}}\right)$ yields, taking account of (32) and (34)

$$
\beta_{i}^{\varepsilon}=0\left(\frac{1}{\left(\lambda^{\varepsilon}\right)^{N-2}}+\varepsilon \log \lambda^{\varepsilon}\right)
$$

From $\left(E_{\lambda i}\right),(35)(36)(40)$ and (42) we deduce that

$$
\begin{array}{r}
\Gamma_{4} \frac{\varepsilon}{\lambda_{i}^{\varepsilon}}-\Gamma_{5}\left(\frac{H\left(x_{i}^{\varepsilon}, x_{i}^{\varepsilon}\right)}{\left(\lambda_{i}^{\varepsilon}\right)^{N-2}}-\sum_{j \neq i} \frac{G\left(x_{i}^{\varepsilon}, x_{j}^{\varepsilon}\right)}{\left(\lambda_{i}^{\varepsilon}\right)^{N / 2}\left(\lambda_{j}^{\varepsilon}\right)^{\frac{N-2}{2}}}\right) \\
=0\left(\frac{1}{\lambda^{\varepsilon}}\left(\frac{\log \lambda^{\varepsilon}}{\left(\lambda^{\varepsilon}\right)^{N}}+\varepsilon^{2} \log \lambda^{\varepsilon}\right)\right)
\end{array}
$$

Let us perform the change of variables

$$
\frac{1}{\lambda_{i}^{\frac{N-2}{2}}}=\left(\frac{\Gamma_{4}}{\Gamma_{5}}\right)^{1 / 2} \Lambda_{i} \varepsilon^{1 / 2}
$$

Note that the properties

$$
\lambda_{i}^{\varepsilon} \rightarrow+\infty, \varepsilon \log \lambda_{i}^{\varepsilon} \rightarrow 0, \frac{\lambda_{i}^{\varepsilon}}{\lambda_{j}^{\varepsilon}}<c_{0}
$$

are translated in

$$
\Lambda_{i}^{\varepsilon} \varepsilon^{1 / 2} \rightarrow 0, \varepsilon \log \Lambda_{i}^{\varepsilon} \rightarrow 0, \frac{\Lambda_{i}^{\varepsilon}}{\Lambda_{j}^{\varepsilon}}<c_{0}
$$

and that (43) reads as

$$
\begin{aligned}
& 1-H\left(x_{i}^{\varepsilon}, x_{i}^{\varepsilon}\right)\left(\Lambda_{i}^{\varepsilon}\right)^{2}+\sum_{j \neq i} G\left(x_{i}^{\varepsilon}, x_{j}^{\varepsilon}\right) \Lambda_{i}^{\varepsilon} \Lambda_{j}^{\varepsilon} \\
& =0\left(\left(\Lambda^{\varepsilon}\right)^{2}\left(\Lambda^{\varepsilon} \varepsilon^{1 / 2}\right)^{\frac{4}{N-2}} \log \left(\Lambda^{\varepsilon} \varepsilon^{1 / 2}\right)+\varepsilon\left|\log \Lambda^{\varepsilon} \varepsilon^{1 / 2}\right|\right) \\
& =0\left(\left|\Lambda^{\varepsilon}\right|^{2}+1\right)
\end{aligned}
$$

Dividing each of these equalities by $\Lambda_{i}^{\varepsilon}$ we get

$$
M\left(\mathrm{x}^{\varepsilon}\right)^{t} \Lambda^{\varepsilon}+\circ\left(\left|\Lambda^{\varepsilon}\right|\right)={ }^{t}\left(\frac{1}{\Lambda^{\varepsilon}}\right)+\circ\left(\frac{1}{\left|\Lambda^{\varepsilon}\right|}\right)
$$

with

$$
\Lambda=\left(\Lambda_{1}, \cdots, \Lambda_{k}\right), \frac{1}{\Lambda}=\left(\frac{1}{\Lambda_{1}}, \cdots, \frac{1}{\Lambda_{k}}\right)
$$

The scalar product of (45) with $r\left(\mathrm{x}^{\varepsilon}\right)$ yields

$$
\rho\left(\mathbf{x}^{\varepsilon}\right) r\left(\mathbf{x}^{\varepsilon}\right) \cdot{ }^{t} \Lambda^{\varepsilon}+\circ\left(\left|\Lambda^{\varepsilon}\right|\right)=r\left(\mathbf{x}^{\varepsilon}\right) \cdot{ }^{t}\left(\frac{1}{\Lambda^{\varepsilon}}\right)+\circ\left(\frac{1}{\left|\Lambda^{\varepsilon}\right|}\right)
$$


On the other hand, we deduce from $\left(E_{x_{i}}\right)$, through (37) (38) (41) (42), that

$$
\frac{\partial H}{\partial a}\left(x_{i}^{\varepsilon}, x_{i}^{\varepsilon}\right) \Lambda_{i}^{\varepsilon}-\sum_{j \neq i} \frac{\partial G}{\partial a}\left(x_{i}^{\varepsilon}, x_{j}^{\varepsilon}\right) \Lambda_{j}^{\varepsilon}
$$

$=0\left(\frac{\varepsilon^{\frac{N-3}{N-2}}}{\left|\Lambda^{\varepsilon}\right|^{\frac{N}{N-2}}}+\left(\left|\Lambda^{\varepsilon}\right|^{2} \varepsilon^{1 / 2} \quad\right.\right.$ if $\quad N=4 ;\left|\Lambda^{\varepsilon}\right|^{\frac{N+2}{N-2}} \varepsilon^{\frac{2}{N-2}} \log \left(\left|\Lambda^{\varepsilon}\right| \varepsilon^{1 / 2}\right) \quad$ if $\left.\left.\quad N \geq 5\right)\right)$

We distinguish three cases

- 1) $\Lambda^{\varepsilon} \rightarrow 0$

- 2) $\Lambda^{\varepsilon} \rightarrow \bar{\Lambda} \in\left(\mathbf{R}_{+}^{*}\right)^{4}$

- 3) $\Lambda^{\varepsilon} \rightarrow+\infty\left(\Lambda_{i}^{\varepsilon} \rightarrow+\infty, \forall i\right)$

From (46) we see that case 1) cannot occur, since in this case the left hand side would go to zero (remember that $\rho$ is bounded from above on $\Omega_{d_{0}}^{k}$ and $|r(\mathrm{x})|=1$ ), and the right hand side to infinity. Let us consider the second case. Denoting by $\overline{\mathrm{x}} \in \Omega_{d_{0}}^{k}$ the limit of $\left(\mathrm{x}^{\varepsilon}\right)$ (up to a subsequence), from (46) we obtain

$$
\rho(\overline{\mathbf{x}}) r(\overline{\mathbf{x}})^{t} \bar{\Lambda}=r(\overline{\mathbf{x}}) \cdot{ }^{t}\left(\frac{1}{\bar{\Lambda}}\right)
$$

Hence $\rho(\overline{\mathbf{x}})>0$. Moreover, passing to the limit in (45), we get

$$
M(\overline{\mathrm{x}}) \bar{\Lambda}=\frac{1}{\bar{\Lambda}}
$$

This means that $\bar{\Lambda}$ is a critical point of $F_{\overline{\mathbf{x}}}$ on $\left(\mathbf{R}_{+}^{*}\right)^{k}$, i.e. $\bar{\Lambda}=\Lambda(\overline{\mathbf{x}})$ according to our notations. Then, the limit in (47) yields

$$
\frac{\partial H}{\partial a}\left(\bar{x}_{i}, \bar{x}_{i}\right) \Lambda_{i}(\overline{\mathbf{x}})-\sum_{j \neq i} \frac{\partial G}{\partial a}\left(\bar{x}_{i}, \bar{x}_{j}\right) \Lambda_{j}(\overline{\mathbf{x}})=0
$$

This may be written

$$
\frac{\partial H}{\partial \mathbf{x}_{i}}(\overline{\mathbf{x}}) \cdot{ }^{t} \Lambda(\overline{\mathbf{x}})=0
$$

implying

$$
\frac{\partial \tilde{F}}{\partial x_{i}}(\overline{\mathbf{x}})=0
$$

i.e. exactly what we wanted to prove. Let us now consider the last case. Still denoting by $\overline{\mathbf{x}}$ the limit of $\left(\mathbf{x}^{\varepsilon}\right),(46)$ shows that $\rho\left(\mathbf{x}^{\varepsilon}\right)=0\left(\frac{1}{\left|\Lambda^{\varepsilon}\right|}\right)$ and then $\rho(\overline{\mathbf{x}})=0$. (45) gives

$$
M\left(\mathrm{x}^{\varepsilon}\right) \Lambda^{\varepsilon}=o\left(\left|\Lambda^{\varepsilon}\right|\right)
$$

so that $\Lambda^{\varepsilon}$ may be written under the form

$$
\Lambda^{\varepsilon}=\beta^{\varepsilon} r\left(\mathrm{x}^{\varepsilon}\right)+\bar{r}^{\varepsilon}
$$


with $\beta^{\varepsilon} \rightarrow+\infty, \bar{r}^{\varepsilon} \cdot r\left(\mathrm{x}^{\varepsilon}\right)=0$, and $\left|\bar{r}^{\varepsilon}\right|=0\left(\beta^{\varepsilon}\right)$. This follows from a decomposition of $\Lambda^{\varepsilon}$ on a basis of eigenvectors for $M\left(\mathrm{x}^{\varepsilon}\right)$; since $\rho(\mathrm{x})$ is simple, all the other eigenvalues remain bounded from below far from zero as $\rho\left(\mathbf{x}^{\varepsilon}\right) \rightarrow 0$. (47) leads to

$$
\frac{\partial M}{\partial x_{i}}\left(\mathrm{x}^{\varepsilon}\right) \cdot{ }^{t} \Lambda^{\varepsilon}=o\left(\left|\Lambda^{\varepsilon}\right|\right)
$$

which means, through (48), that

$$
\beta^{\varepsilon} \frac{\partial M}{\partial x_{i}}\left(\mathbf{x}^{\varepsilon}\right) \cdot{ }^{t} r\left(\mathbf{x}^{\varepsilon}\right)+\frac{\partial M}{\partial x_{i}}\left(\mathbf{x}^{\varepsilon}\right) \cdot{ }^{t} \bar{r}^{\varepsilon}=o\left(\beta^{\varepsilon}\right)
$$

The matrix $\frac{\partial M}{\partial x_{i}}(\mathbf{x})$ being bounded on the set $\left\{\mathbf{x} \in \Omega^{k} d_{0} / \rho \geq \rho_{0}\right\}$ for any $\rho_{0} \in \mathbb{R}$, we get

$$
\frac{\partial M}{\partial x_{i}}\left(\mathbf{x}^{\varepsilon}\right) \cdot{ }^{t} r\left(\mathbf{x}^{\varepsilon}\right)=o(1)
$$

Let us consider the equality

$$
M(\mathrm{x}) \cdot{ }^{t} r(\mathrm{x})=\rho(\mathrm{x})^{t} r(\mathrm{x})
$$

and derivate it with respect to $x_{i}$; we obtain

$$
\frac{\partial M}{\partial x_{i}}(\mathbf{x}) \cdot{ }^{t} r(\mathbf{x})+M(\mathbf{x}) \cdot \frac{\partial^{t} r}{\partial x_{i}}(\mathbf{x})=\frac{\partial \rho}{\partial x_{i}}(\mathbf{x})^{t} r(\mathbf{x})+\rho(\mathbf{x}) \frac{\partial^{t} r}{\partial x_{i}}(\mathbf{x})
$$

The scalar product with $r(\mathbf{x})$ gives

$$
r(\mathrm{x}) \cdot \frac{\partial M}{\partial x_{i}}(\mathrm{x}) \cdot{ }^{t} r(\mathrm{x})=\frac{\partial \rho}{\partial x_{i}}(\mathrm{x})
$$

since $|r(\mathbf{x})|=1$ and $r(\mathbf{x}) \cdot \frac{\partial^{t} r(\mathbf{x})}{\partial x_{i}}=0$. Therefore (49) yields

$$
\frac{\partial \rho}{\partial x_{i}}\left(\mathbf{x}^{\varepsilon}\right)=o(1)
$$

and so

$$
\frac{\partial \rho}{\partial x_{i}}(\overline{\mathbf{x}})=0
$$

This concludes the proof of the first part of Theorem 1. Furthermore, we see that if 0 is a regular value for $\rho$, the last case that we considered cannot occur, and even that there exists $\rho_{0}>0$ such that $\rho(\bar{x}) \geq \rho_{0}$. Indeed, there would exist otherwise a sequence $\left(\mathrm{x}^{n}\right)$ in $\Omega_{d_{0}}^{k}$ such that $\rho\left(\mathrm{x}^{n}\right)>0, \rho\left(\mathrm{x}^{n}\right) \rightarrow 0, \tilde{F}^{\prime}\left(\mathrm{x}^{n}\right)=0$. Let $\overline{\mathrm{x}}$ be its limit (up to a subsequence). Taking the scalar with $r\left(\mathrm{x}^{n}\right)$ of the equality

$$
M\left(\mathrm{x}^{n}\right) \cdot{ }^{t} \Lambda\left(\mathrm{x}^{n}\right)={ }^{t}\left(\frac{1}{\Lambda\left(\mathrm{x}^{n}\right)}\right)
$$


we get

$$
\rho\left(\mathbf{x}^{n}\right) r\left(\mathbf{x}^{n}\right) \cdot{ }^{t} \Lambda\left(\mathbf{x}^{n}\right)=r\left(\mathbf{x}^{n}\right) \cdot{ }^{t}\left(\frac{1}{\Lambda\left(\mathbf{x}^{n}\right)}\right)
$$

Then, $\rho\left(\mathrm{x}^{n}\right) \rightarrow 0$ shows that necessarily $\Lambda\left(\mathrm{x}^{n}\right) \rightarrow+\infty$. Therefore, we can repeat the previous argument (case 3$)$ ), which leads to

$$
\rho(\overline{\mathbf{x}})=0, \rho^{\prime}(\overline{\mathbf{x}})=0
$$

in contradiction with the assumption that 0 is not a critical value for $\rho$; hence the announced result. Lastly, a simple computation, taking account of (6) (24) (26) (31) leads in the case where $\rho(\overline{\mathbf{x}})>0$ to the following expansion for $J_{\varepsilon}\left(u_{\varepsilon}\right)$ :

$$
J_{\varepsilon}\left(u_{\varepsilon}\right)=c_{k}+k \gamma_{1} \varepsilon|\log \varepsilon|+k \gamma_{2} \varepsilon+2 \gamma_{1} \varepsilon \tilde{F}(\overline{\mathbf{x}})+\circ(\varepsilon)
$$

where $\gamma_{1}=\frac{\Gamma_{4}}{N-2}>0$ and $\gamma_{2}=-\frac{\Gamma_{4}}{N-2} \log \frac{\Gamma_{4}}{\Gamma_{5}}$ are constants which depend on $N$ only, and $\overline{\mathrm{x}} \in \Omega_{d_{0}}^{k}$ is a critical point of $\tilde{F}$.

\section{Remark :}

For $\mathrm{x} \in \Omega_{d_{0}}^{k}$, we have

$$
\frac{\Lambda_{i}(\mathrm{x})}{\Lambda_{j}(\mathrm{x})} \leq \bar{\Lambda} \quad \forall i, j
$$

where $\bar{\Lambda}$ is a constant which depends on $\Omega, d_{0}$ only. Indeed, on $\Omega_{d_{0}}^{k}, H(x, x)$ is bounded, and on $\Omega_{d_{0}}^{k} \times \Omega_{d_{0}}^{k}, G(x, y)$ remains far from zero. Therefore, the equality

$$
M(\mathrm{x}) \cdot{ }^{t} \Lambda(\mathrm{x})={ }^{t}\left(\frac{1}{\Lambda(\mathrm{x})}\right)
$$

which implies

$$
H\left(x_{j}, x_{j}\right) \Lambda_{j}(\mathrm{x}) \geq G\left(x_{i}, x_{j}\right) \Lambda_{i}(\mathrm{x}) \quad \forall_{i, j}, i \neq j
$$

leads to

$$
\frac{\Lambda_{i}(\mathbf{x})}{\Lambda_{j}(\mathbf{x})} \leq \frac{H\left(x_{j}, x_{j}\right)}{G\left(x_{j}, x_{j}\right)} \leq \frac{\sup _{x \in \Omega_{d_{0}}} H(x, x)}{\inf _{x, y \in \Omega_{d_{0}}} G(x, y)}=\bar{\Lambda}
$$

On another hand, on the relatively compact set in $\Omega^{k}, \rho^{+}\left(d_{0}\right)=\left\{\mathrm{x} \in \Omega_{d_{0}}^{k} / \rho(\mathrm{x})>0\right\}$, there exist $\underline{r}, \bar{r}>0$ such that

$$
\underline{r} \leq r_{i}(\mathrm{x}) \leq \bar{r} \quad \forall i, \forall \mathrm{x} \in \rho^{+}\left(d_{0}\right)
$$

Then we deduce from the equality

$$
r(\mathbf{x}) \cdot M(\mathbf{x}) \cdot{ }^{t} \Lambda(\mathbf{x})=\rho(\mathbf{x}) r(\mathbf{x}) \cdot{ }^{t} \Lambda(\mathbf{x})=r(\mathbf{x}) \cdot{ }^{t}\left(\frac{1}{\Lambda(\mathrm{x})}\right)
$$

the existence of $\underline{\mu}, \bar{\mu}>0$ such that

$$
\underline{\mu} \leq \rho(\mathrm{x}) \Lambda_{i}^{2}(\mathrm{x}) \leq \bar{\mu} \quad \forall i, \forall \mathrm{x} \in \rho^{+}\left(d_{0}\right)
$$


As

$$
\tilde{F}(\mathrm{x})=\frac{k}{2}-\sum_{i=1}^{k} \log \Lambda_{i}(\mathrm{x})
$$

there exist two constants $\underline{\nu}, \bar{\nu}$ such that

$$
\underline{\nu}+\frac{k}{2} \log \rho(\mathrm{x}) \leq \tilde{F}(\mathrm{x}) \leq \bar{\nu}+\frac{k}{2} \log \rho(\mathrm{x}) \quad \forall \mathbf{x} \in \rho^{+}\left(d_{0}\right)
$$

Let us assume that 0 is a regular value for $\rho$, so that there exists $\rho_{0}>0$ such that all the critical points of $\tilde{F}$ in $\rho^{+}\left(d_{0}\right)$ are in fact in the set $\left\{\mathrm{x} \in \Omega_{d_{0}}^{k} / \rho(\mathrm{x}) \geq \rho_{0}\right\}$. This implies that the critical values for $J_{\varepsilon}$ corresponding to eventual $k$-singular solutions as $\varepsilon$ goes to zero are bounded from below for $\varepsilon$ small enough by

$$
d_{\varepsilon}=c_{\varepsilon}-\underline{c} \varepsilon
$$

with

$$
c_{\varepsilon}=c_{k}+k \gamma_{1} \varepsilon|\log \varepsilon|+k \gamma_{2} \varepsilon ; \underline{c}=-2 \gamma_{1}\left(\underline{\nu}+\frac{k}{2} \log \rho_{0}\right)+1
$$

This follows directly from (50).

(Note that these critical values are also bounded from above by $d_{\varepsilon}=c_{\varepsilon}+\bar{c} \varepsilon$, with $\bar{c}=2 \gamma_{1}\left(\bar{\nu}+\frac{k}{2} \log m\right)+1$, and $\left.m=\sup _{\mathbf{x} \in \Omega_{d_{0}}^{k}} \rho(\mathbf{x})\right)$.

We turn now to the converse part of Theorem 1 . Thus we consider a point $\overline{\mathrm{x}}$ in $\Omega^{k}$ such that $\rho(\overline{\mathbf{x}})>0$ and $\overline{\mathrm{x}}$ is a nondegenerate critical point of $\tilde{F}$. We set

$$
\begin{aligned}
& \frac{1}{\lambda_{i}^{\frac{N-2}{2}}}=\left(\frac{\Gamma_{4}}{\Gamma_{5}}\right)^{1 / 2}\left(\Lambda_{i}(\overline{\mathrm{x}})+\zeta_{i}\right) \varepsilon^{1 / 2} \\
& x_{i}=\overline{x_{i}}+\xi_{i}
\end{aligned}
$$

where $\zeta_{i} \in \mathbf{R}$ and $\xi_{i} \in \mathbf{R}^{N}$ are assumed to be small. With these changes of variables, according to the previous computations, the system (E) appears to be equivalent to (with $v=\bar{v}(\varepsilon, \alpha, \lambda, \mathbf{x})$ ) :

$$
\begin{gathered}
\left(2 H\left(\overline{x_{i}}, \overline{x_{i}}\right) \overline{\Lambda_{i}}-\sum_{j \neq i} G\left(\overline{x_{i}}, \overline{x_{j}}\right) \overline{\Lambda_{j}}\right) \zeta_{i}-\sum_{j \neq i} G\left(\overline{x_{i}}, \overline{x_{j}}\right) \overline{\Lambda_{i}} \zeta_{j} \\
+\left(2 \frac{\partial H}{\partial a}\left(\overline{x_{i}}, \overline{x_{i}}\right){\overline{\Lambda_{i}}}^{2}-\sum_{j \neq i} \frac{\partial G}{\partial a}\left(\overline{x_{i}}, \overline{x_{j}}\right) \overline{\Lambda_{i}}\right) \xi_{i}-\sum_{j \neq i} \frac{\partial G}{\partial b}\left(\overline{x_{i}}, \overline{x_{j}}\right) \overline{\Lambda_{i} \Lambda_{j}} \xi_{j} \\
=V_{\lambda_{i}}(\varepsilon, \beta, \zeta, \xi)
\end{gathered}
$$




$$
\begin{gathered}
\frac{\partial H}{\partial a}\left(\overline{x_{i}}, \overline{x_{i}}\right) \zeta_{i}-\sum_{j \neq i} \frac{\partial G}{\partial a}\left(\overline{x_{i}}, \overline{x_{j}}\right) \zeta_{j} \\
+\left(\frac{\partial^{2} H}{\partial a^{2}}\left(\overline{x_{i}}, \overline{x_{i}}\right) \overline{\Lambda_{i}}+\frac{\partial^{2} H}{\partial a \partial b}\left(\overline{x_{i}}, \overline{x_{i}}\right) \overline{\Lambda_{i}}-\sum_{j \neq i} \frac{\partial^{2} G}{\partial a^{2}}\left(\overline{x_{i}}, \overline{x_{j}}\right) \overline{\Lambda_{j}}\right) \xi_{i} \\
-\sum_{j \neq i} \frac{\partial^{2} G}{\partial a \partial b}\left(\overline{x_{i}}, \overline{x_{j}}\right) \overline{\Lambda_{j}} \xi_{i} \\
=V_{x_{i}}(\varepsilon, \beta, \zeta, \xi)
\end{gathered}
$$

where $V_{\alpha_{i}}, V_{\lambda_{i}}, V_{x_{i}}$ are smooth functions which satisfy

$$
\left\{\begin{array}{l}
V_{\alpha_{i}}=0\left(\varepsilon|\log \varepsilon|+|\beta|^{2}\right) \\
V_{\lambda_{i}}=0\left(\varepsilon^{\frac{2}{N-2}}|\log \varepsilon|+|\beta|+|\zeta|^{2}+|\xi|^{2}\right) \\
V_{x_{i}}=0\left(\left(\varepsilon^{1 / 2} \text { if } N=4 ; \varepsilon^{\frac{2}{N-2}}|\log \varepsilon| \text { if } N \geq 5\right)+|\beta|+|\zeta|^{2}+|\xi|^{2}\right)
\end{array}\right.
$$

This system may also be written

$$
\left\{\begin{aligned}
\beta & =V(\varepsilon, \beta, \zeta, \xi) \\
L(\zeta, \xi) & =W(\varepsilon, \beta, \zeta, \xi)
\end{aligned}\right.
$$

where $L$ is fixed linear operator of $\mathbf{R}^{k(N+1)}$ and $V, W$ are smooth function satisfying (59) $\left\{\begin{array}{l}V(\varepsilon, \beta, \zeta, \xi)=0\left(\varepsilon|\log \varepsilon|+|\beta|^{2}\right) \\ W(\varepsilon, \beta, \zeta, \xi)=0\left(\left(\varepsilon^{1 / 2} \text { if } N=4 ; \varepsilon^{\frac{2}{N-2}}|\log \varepsilon| \text { if } N \geq 5\right)+|\beta|+|\zeta|^{2}+|\xi|^{2}\right)\end{array}\right.$

Moreover, a simple computation shows that the determinant of $L$ is proportionnal to the determinant of $\tilde{F}^{\prime \prime}(\overline{\mathrm{x}})$. $\quad \overline{\mathrm{x}}$ being a nondegenerate critical point of $\tilde{F}$ by assumption, $L$ is invertible, and Brouwer's fixed point theorem shows that (58) has a solution $\left(\beta^{\varepsilon}, \zeta^{\varepsilon}, \xi^{\varepsilon}\right)$ for $\varepsilon$ small enough, such that

$$
\begin{aligned}
& \left|\beta^{\varepsilon}\right|=0(\varepsilon|\log \varepsilon|) \\
& \left|\zeta^{\varepsilon}\right|=0\left(\varepsilon^{1 / 2} \text { if } N=4 ; \varepsilon^{\frac{2}{N-2}}|\log \varepsilon| \text { if } N \geq 5\right) \\
& \left|\xi^{\varepsilon}\right|=0\left(\varepsilon^{1 / 2} \text { if } N=4 ; \varepsilon^{\frac{2}{N-2}}|\log \varepsilon| \text { if } N \geq 5\right)
\end{aligned}
$$

By construction, the corresponding $u_{\varepsilon} \in H_{0}^{1}(\Omega)$ is a critical point of $J_{\varepsilon}$, i.e. $u_{\varepsilon}$ satisfies on $\Omega$

$$
-\Delta u_{\varepsilon}=\left|u_{\varepsilon}\right|^{p-1} u_{\varepsilon}
$$

Multiplying this equation by $u_{\varepsilon}^{-}=\max \left(0,-u_{\varepsilon}\right)$ and integrating on $\Omega$, we get

$$
\int_{\Omega}\left|\nabla u_{\varepsilon}^{-}\right|^{2}=\int_{\Omega}\left(u_{\varepsilon}^{-}\right)^{p+1-\varepsilon}
$$


On the other hand, the Sobolev embedding theorem yields

$$
\left(\int_{\Omega}\left(u_{\varepsilon}^{-}\right)^{p+1-\varepsilon}\right)^{\frac{2}{p+1-\varepsilon}} \leq C \int_{\Omega}\left|\nabla u_{\varepsilon}^{-}\right|^{2}
$$

(61) and (62) imply that either $u_{\varepsilon}^{-} \equiv 0$, or $\left|u_{\varepsilon}^{-}\right|_{p+1-\varepsilon}$ is far from zero. As $\left|u_{\varepsilon}^{-}\right|_{p+1-\varepsilon} \leq\left|\bar{v}^{\varepsilon}\right|_{p+1-\varepsilon}$ and $\bar{v}^{\varepsilon}$ goes to zero in $H_{0}^{1}(\Omega)$, we obtain that $u_{\varepsilon}^{-} \equiv 0$ for $\varepsilon$ small enough. Since $u_{\varepsilon}$ is a nontrivial positive solution of (59), the strong maximum principle ensures that $u_{\varepsilon}>0$ on $\Omega$, and then $u_{\varepsilon}$ is a solution to $\left(P_{\varepsilon}\right)$, which blows-up at $\bar{x}_{1}, \cdots, \bar{x}_{k}$ as $\varepsilon$ goes to zero. The index of this solution as a critical point of $J_{\varepsilon}$ is $\ell+k$, where $\ell$ is the index of $\overline{\mathrm{x}}$ as a critical point of $\tilde{F}$. Indeed, considering $K_{\varepsilon}$, the procedures that we followed consisted firsteval in minimizing with respect to $v$, then maximizing with respect to the $\alpha_{i}^{\prime} s$ (contribution $k$ to the index), minimizing with respect to the $\lambda_{i}^{\prime} s$ and lastly in taking $\mathrm{x}$ as a critical point of a $C^{2}$-perturbation of $\tilde{F}$ in a neighborhood of $\overline{\mathrm{x}}$ (contribution $\ell$ to the index). One could get directly convinced by these arguments in computing the second derivatives of $K_{\varepsilon}$, as in [15] ; as a byproduct we obtain that the $k$-singular solution $u_{\varepsilon}$ to $\left(P_{\varepsilon}\right)$ corresponding to $\overline{\mathrm{x}}$ is unique. These arguments also apply to the solutions of $\left(P_{\varepsilon}\right)$ that we considered in the first part of the theorem, except that the index may be larger than $\ell+k$ in the case where $\mathbf{x}$ is a degenerate critical point of $\widetilde{F}$ or a critical point of $\rho$; nevertheless, the index is bounded from above by $N k+k=(N+1) k$.

\section{Proof of Theorem 2}

From now on, we assume that 0 is a regular value for $\rho$.

Let $\eta$ be a fixed number, $0<\eta<\frac{S^{N / 2}}{N}$. Our aim in this last section is to compute the contribution to the relative topology of $J_{\varepsilon}^{c_{k}+\eta}$ with respect to $J_{\varepsilon}^{c_{k}-\eta}$ of the $k$-singular solutions to $\left(P_{\varepsilon}\right)$ that we studied before. From $(52)$ we know that, in fact, we can compute this relative topology between the levels $c_{k}+\eta$ and $c_{\varepsilon}-c \varepsilon$, with $c \geq \underline{c}$ and $\underline{c}$ given by (53). Assume that $\mathcal{V}_{\varepsilon}$ is an open neighborhood of the eventual $k$-singular solutions to $\left(P_{\varepsilon}\right)$ such that, on the boundary of $\mathcal{V}_{\varepsilon}$, either $-J_{\varepsilon}^{\prime}$ is pointing inward $\mathcal{V}_{\varepsilon}$, or $J_{\varepsilon}$ is less than $c_{\varepsilon}-c \varepsilon$.

Thus, what we want to compute is exactly the relative topology

$$
\left(J_{\varepsilon}^{c_{k}+\eta} \cap \mathcal{V}_{\varepsilon}, J_{\varepsilon}^{c_{\varepsilon}-c \varepsilon} \cap \mathcal{V}_{\varepsilon}\right)
$$

It remains to define $c$ and $\mathcal{V}_{\varepsilon}$ in a suitable way, in order to satisfy the previous conditions and to make the computation possible. We define successively some quantities whose choice will appear in the following. Thus, we set

$$
\left\{\begin{array}{cll}
\rho_{1} & \text { s.t. } & \rho \text { has no critical value in }\left[0, \rho_{1}\right] \\
c_{1}>\max (0, \underline{c}) & \text { s.t. } & 2 \gamma_{1} \tilde{F}(\mathrm{x}) \leq-\frac{c_{1}}{4} \text { implies } \rho(\mathrm{x}) \leq \rho_{1} \\
\rho_{2} & \text { s.t. } & \rho(\mathrm{x}) \leq \rho_{2} \text { implies } 2 \gamma_{1} \tilde{F}(\mathrm{x}) \leq-4 c_{1} \\
c_{2} & \text { s.t. } & 2 \gamma_{1} \tilde{F}(\mathrm{x}) \leq-\frac{c_{2}}{4} \text { implies } \rho(\mathrm{x}) \leq \rho_{2} \\
\rho_{3} & \text { s.t. } & \rho(\mathrm{x}) \leq \rho_{3} \text { implies } 2 \gamma_{1} \tilde{F}(\mathrm{x}) \leq-4 c_{2}
\end{array}\right.
$$


$\mathrm{x}$ being assumed to be in $\Omega_{d_{0}}^{k}$. The existence of quantities verifying the listed conditions follows from (51). They will allow us to include level sets of $\tilde{F}$ between level sets of $\rho$.

Now, we choose $\Lambda_{0}>0$ small enough so that for any $\mathrm{x} \in\left\{\mathrm{x} \in \Omega_{d_{0}}^{k} / \rho(\mathrm{x})>\rho_{3}\right\}$ and $|\Lambda-\Lambda(\mathrm{x})|=\left(\sum_{i=1}^{k}\left(\Lambda_{i}-\Lambda_{i}(\mathrm{x})\right)^{2}\right)^{1 / 2}<\Lambda_{0}$, we have

$$
\left\{\begin{array}{lll}
2 \gamma_{1} F_{\mathbf{x}}(\Lambda) \leq-\frac{c_{1}}{2} & \text { implies } 2 \gamma_{1} \tilde{F}(\mathbf{x}) \leq-\frac{c_{1}}{4} \\
2 \gamma_{1} F_{\mathbf{X}}(\Lambda) & \leq-\frac{c_{2}}{2} & \text { implies } 2 \gamma_{1} \tilde{F}(\mathbf{x}) \leq-\frac{c_{2}}{4} \\
2 \gamma_{1} \tilde{F}(\mathbf{x}) & \leq-4 c_{1} & \text { implies } 2 \gamma_{1} F_{\mathbf{x}}(\Lambda) \leq-2 c_{1} \\
2 \gamma_{1} \tilde{F}(\mathbf{x}) \leq-4 c_{2} & \text { implies } 2 \gamma_{1} F_{\mathbf{x}}(\Lambda) \leq-4 c_{2}
\end{array}\right.
$$

Note that for $\mathrm{x} \in \bar{\Omega}_{d_{0}}^{k}, \rho(\mathrm{x})>0$, and $d\left(x_{i}, \partial \Omega\right)=d_{0}$, we have for $d_{0}$ small enough

$$
\frac{\partial \tilde{F}}{\partial x_{i}}(\mathrm{x}) \cdot n_{i} \geq \kappa\left(d_{0}\right)>0
$$

where $n_{i}$ denotes the outward normal to $\partial \Omega_{d_{0}}$ at $x_{i}$. For a proof of this fact, we refer to Appendix B. Then, we choose $\Lambda_{0}$ small enough so that we have also, for same $\mathrm{x}$ and $|\Lambda-\Lambda(\mathrm{x})|<\Lambda_{0}$

$$
\frac{\partial}{\partial x_{i}} F_{\mathbf{x}}(\Lambda) \cdot n_{i} \geq \kappa^{\prime}\left(d_{0}\right)>0
$$

Lastly, we remark that

$$
(\Lambda-\Lambda(\mathrm{x})) \cdot \frac{\partial}{\partial \Lambda} F_{\mathrm{x}}(\Lambda)=(\Lambda-\Lambda(\mathrm{x})) \cdot M(\mathrm{x}) \cdot{ }^{t}(\Lambda-\Lambda(\mathrm{x}))+\sum_{i=1}^{k} \frac{\left(\Lambda_{i}-\Lambda_{i}(\mathrm{x})\right)^{2}}{\Lambda_{i} \Lambda_{i}(\mathrm{x})}
$$

$$
\geq \rho(\mathbf{x})|\Lambda-\Lambda(\mathbf{x})|^{2}
$$

Then, our aim turns out to be the computation of the relative topology

$$
\begin{aligned}
& \left(J_{\varepsilon}^{c_{k}+\eta} \cap \mathcal{V}_{\varepsilon}, J_{\varepsilon}^{c_{\varepsilon}-c_{1} \varepsilon} \cap \mathcal{V}_{\varepsilon}\right) \\
= & \left(K_{\varepsilon}^{c_{k}+\eta} \cap V_{\varepsilon}, K_{\varepsilon}^{c_{\varepsilon}-c_{1} \varepsilon} \cap V_{\varepsilon}\right)
\end{aligned}
$$

where $V_{\varepsilon}$ and $\mathcal{V}_{\varepsilon}$ are corresponding through the diffeomorphism described in Section 2 , and

$$
\begin{aligned}
V_{\varepsilon}= & \left\{(\alpha, \lambda, \mathbf{x}, v) /\left|\alpha_{i}-\alpha\right|<\alpha_{0}, d\left(x_{i}, \partial \Omega\right)>d_{0}, \forall i ; \rho(\mathbf{x})>\rho_{3},\right. \\
& |\Lambda-\Lambda(\mathbf{x})|<\Lambda_{0}, v \in E_{\lambda, \mathbf{x}}, \quad \text { and } \\
& \left.|v-\bar{v}(\varepsilon, \alpha, \lambda, \mathbf{x})|<\nu_{0}\left(\varepsilon \text { if } N<6 ; \varepsilon|\log \varepsilon| \text { if } N=6 ; \varepsilon^{\frac{N+2}{2(N-2)}} \text { if } N>6\right)\right\}
\end{aligned}
$$


The link between $\lambda$ and $\Lambda$ is, as stated previously

$$
\frac{1}{\lambda_{i}^{\frac{N-2}{2}}}=\left(\frac{\Gamma_{4}}{\Gamma_{5}}\right)^{1 / 2} \Lambda_{i} \varepsilon^{1 / 2}
$$

and $\bar{v}$ is defined by Proposition 2. From Section 3 we known that $V_{\varepsilon}$ is a neighborhood of the eventual $k$-singular critical points of $K_{\varepsilon}$ we are interested in. The constants $\alpha_{0}$ and $\nu_{0}$ are chosen in the following way :

We take $\nu_{0}$ large enough so that we have, for $(\alpha, \lambda, \mathbf{x}, v) \in \overline{\mathcal{V}}_{\varepsilon},|v-\bar{v}|_{H_{0}^{1}}=\nu_{0}(\varepsilon$ if $N<6 ; \varepsilon|\log \varepsilon|$ if $N>6$ ), and $\varepsilon$ small enough

$$
(v-\bar{v}) \cdot \frac{\partial K_{\varepsilon}}{\partial v}(\alpha, \lambda, \mathbf{x}, v)>0
$$

We take $\alpha_{0}$ small enough so that, for $(\alpha, \lambda, \mathrm{x}, v) \in \overline{\mathcal{V}}_{\varepsilon}$ and $\varepsilon$ small enough, we have

$$
\begin{gathered}
\frac{\partial K_{\varepsilon}}{\partial x_{i}} \cdot n_{i}>0 \quad \text { if } \quad d\left(x_{i}, \partial \Omega\right)=d_{0} \\
(\Lambda-\Lambda(\mathrm{x})) \cdot \frac{\partial K_{\varepsilon}}{\partial \Lambda}>0 \quad \text { if } \quad|\Lambda-\Lambda(\mathrm{x})|=\Lambda_{0}
\end{gathered}
$$

Such a choice of $\nu_{0}$ is made possible by Proposition 2, the existence of $\alpha_{0}$ follows from (37) (65) and (35) (66).

Lastly, let us remark that (32) implies, for $(\alpha, \lambda, \mathrm{x}, v) \in \overline{\mathcal{V}}_{\varepsilon},\left|\alpha_{i}-\alpha\right|=\alpha_{0}$ and $\varepsilon$ small enough

$$
K_{\varepsilon}(\alpha, \lambda, \mathrm{x}, v) \leq c_{\varepsilon}-c_{2} \varepsilon
$$

If $\rho(\mathbf{x})=\rho_{3}$, then by (63) and (64), $2 \gamma_{1} F_{\mathbf{x}}(\Lambda) \leq-2 c_{2}$. As an expansion of $K_{\varepsilon}$ for $(\alpha, \lambda, \mathbf{x}, v) \in \overline{\mathcal{V}}_{\varepsilon}$ yields

$$
K_{\varepsilon}(\alpha, \lambda, \mathrm{x}, v) \leq c_{\varepsilon}+2 \gamma_{1} F_{\mathrm{x}}(\Lambda)+\circ(\varepsilon)
$$

$\rho(\mathbf{x})=\rho_{3}$ implies that

$$
K_{\varepsilon}(\alpha, \lambda, \mathrm{x}, v) \leq c_{\varepsilon}-c_{2} \varepsilon
$$

provided that $\varepsilon$ is suffiently small.

As a consequence of the definitions and properties that we gave, we see that we reached our goal to construct a neighborhood $V_{\varepsilon}$ of the $k$-singular critical points, on the boundary of which either $-K_{\varepsilon}^{\prime}$ is pointing inward $V_{\varepsilon}$, or $K_{\varepsilon}$ is less than $c_{\varepsilon}-c_{1} \varepsilon$ : $K_{\varepsilon}$ is even less than $c_{\varepsilon}-c_{2} \varepsilon$ 
We turn now to the computation of the relative topology (67). The first step is concerned with the $v$-variable. We set

$$
\widetilde{K_{\varepsilon}}(\alpha, \lambda, \mathbf{x})=K_{\varepsilon}(\alpha, \lambda, \mathbf{x}, \bar{v})
$$

for $(\alpha, \lambda, \mathbf{x})$ in

$$
\tilde{V}_{\varepsilon}=\left\{(\alpha, \lambda, \mathbf{x}) /\left|\alpha_{i}-\alpha\right|<\alpha_{0}, d\left(x_{i}, \partial \Omega\right)>d_{0}, \forall i ; \rho(\mathbf{x})>\rho_{3},|\Lambda-\Lambda(\mathbf{x})|<\Lambda_{0}\right\}
$$

From Proposition 2 and Morse theory we deduce, since $\bar{v}$ is a strict minimizer of $K_{\varepsilon}$ in a fixed neighborhood of $v=0$, that

$$
\begin{aligned}
K_{\varepsilon}^{c_{k}+\eta} \cap V_{\varepsilon}= & V_{\varepsilon} \\
= & \left\{(\alpha, \lambda, \mathbf{x}, v) /(\alpha, \lambda, \mathbf{x}) \in \tilde{V}_{\varepsilon},\right. \\
& \left.v \in E_{\lambda, \mathbf{x}} \cap D_{H_{0}^{1}}\left(\bar{v}, \nu_{0}\left(\varepsilon \text { if } N<6 ; \varepsilon|\log \varepsilon| \text { if } N=6 ; \varepsilon^{\frac{N+2}{(N-2)}} \text { if } N>6\right)\right)\right\}
\end{aligned}
$$

and

$$
K_{\varepsilon}^{c_{\varepsilon}-c_{1} \varepsilon} \cap V_{\varepsilon}=\left\{(\alpha, \lambda, \mathbf{x}, v) /(\alpha, \lambda, \mathbf{x}) \in \widetilde{V}_{\varepsilon}, \widetilde{K}_{\varepsilon}(\alpha, \lambda, \mathbf{x}) \leq c_{\varepsilon}-c_{1} \varepsilon, v \in D(\alpha, \lambda, \mathbf{x})\right\}
$$

where $D(\alpha, \lambda, \mathbf{x})$ is a subset of $E_{\lambda, \mathbf{x}}$ topologically equivalent to a disk. Therefore

$$
\left(K_{\varepsilon}^{c_{k}+\eta} \cap V_{\varepsilon}, K_{\varepsilon}^{c_{\varepsilon}-c_{1} \varepsilon} \cap V_{\varepsilon}\right) \simeq\left(\tilde{V}_{\varepsilon}, \widetilde{K}_{\varepsilon}^{c_{\varepsilon}-c_{1} \varepsilon} \cap \tilde{V}_{\varepsilon}\right)
$$

The next step consists in defining

$$
\widetilde{\widetilde{K}}_{\varepsilon}=\widetilde{K}_{\varepsilon}(\bar{\alpha}, \lambda, \mathbf{x})
$$

for $(\lambda, \mathbf{x})$ in

$$
\tilde{\tilde{V}}_{\varepsilon}=\left\{(\lambda, \mathbf{x}) / d\left(x_{i}, \partial \Omega\right)>d_{0}, \forall i ; \rho(\mathbf{x})>\rho_{3},|\Lambda-\Lambda(\mathbf{x})|<\Lambda_{0}\right\}
$$

and $\bar{\alpha}=\alpha(\lambda, \mathbf{x})$ is such that $\frac{\partial \tilde{K}_{\varepsilon}}{\partial \alpha}(\bar{\alpha}, \lambda, \mathbf{x})=0$. Such an $\bar{\alpha},\left|\bar{\alpha}_{i}-\alpha\right|<\alpha_{0}$, is unique, and corresponds to a strict and nondegenerate maximum (see (32), and [14] for an estimate of the second derivative of $\widetilde{K_{\varepsilon}}$ with respect to $\alpha$ ). Morse theory yields

$$
\begin{gathered}
{\widetilde{K_{\varepsilon}^{c}}}_{\varepsilon}^{c_{\varepsilon}-c_{1} \varepsilon} \cap \widetilde{V}_{\varepsilon} \\
=\left\{(\alpha, \lambda, \mathbf{x}) \in \widetilde{V}_{\varepsilon} / \widetilde{\widetilde{K}}_{\varepsilon}(\lambda, \mathbf{x}) \leq c_{\varepsilon}-c_{1} \varepsilon, \quad \text { and } \quad \alpha \in D\right\} \\
\cup\left\{(\alpha, \lambda, \mathbf{x}) \in \widetilde{V}_{\varepsilon} / \widetilde{\widetilde{K}}_{\varepsilon}(\lambda, \mathbf{x})>c_{\varepsilon}-c_{1} \varepsilon_{1} \quad \text { and } \quad \alpha \in C(\lambda, \mathbf{x})\right\}
\end{gathered}
$$

Here, $D$ denotes the $k$-square $] \alpha-\alpha_{0}, \alpha+\alpha_{0}\left[{ }^{k}\right.$, topologically equivalent to the unit disk $D^{k}$ of $\mathbf{R}^{k}$, and $C(\lambda, \mathrm{x})$ is equal to $D$ deleted from a subset equivalent to a disk, whose radius goes to zero as $\check{K}_{\varepsilon}(\lambda, \mathrm{x})$ goes to $c_{\varepsilon}-c_{1} \varepsilon$. At the same time

$$
\tilde{V}_{\varepsilon}=\tilde{\widetilde{V}}_{\varepsilon} \times D
$$

Then, we have a natural injective map

$$
\left(\tilde{\tilde{V}}_{\varepsilon}, \widetilde{\widetilde{K}}_{\varepsilon}^{c_{\varepsilon}-c_{1} \varepsilon} \cap \tilde{\tilde{V}}_{\varepsilon}\right) \times\left(D^{k}, S^{k-1}\right) \rightarrow\left(\tilde{V}_{\varepsilon}, \widetilde{K}_{\varepsilon}^{c_{\varepsilon}-c_{1} \varepsilon} \cap \tilde{V}_{\varepsilon}\right)
$$

Since all the critical values for $\widetilde{\widetilde{K}}_{\varepsilon}$ on $\tilde{\tilde{V}}_{\varepsilon}$ are larger than $c_{\varepsilon}-\underline{c} \varepsilon>c_{\varepsilon}-c_{1} \varepsilon$, this map appears to be also surjective, and then is an isomorphism. 
On $\tilde{\tilde{V}}_{\varepsilon}$, a simple computation provides us with the uniform estimate

$$
\widetilde{\widetilde{K}}_{\varepsilon}(\lambda, \mathrm{x})=c_{\varepsilon}+2 \gamma_{1} \varepsilon F_{\mathbf{x}}(\Lambda)+o(\varepsilon)
$$

The way we chose $\rho_{1}, \rho_{2}, c_{1}, c_{2}$ gives us now, together with (69), provides us with the following properties (for $\varepsilon$ sufficiently small)

$$
\begin{aligned}
\widetilde{\widetilde{K}}_{\varepsilon}^{c_{\varepsilon}-c_{2} \varepsilon} \cap \widetilde{\tilde{V}}_{\varepsilon} & \subset\left\{(\lambda, \mathrm{x}) \in \widetilde{\widetilde{V}}_{\varepsilon} / \rho(\mathrm{x})<\rho_{2}\right\} \\
& \subset \widetilde{\widetilde{K}}_{\varepsilon}^{c_{\varepsilon}-c_{1} \varepsilon} \\
& \subset\left\{(\lambda, \mathrm{x}) \in \widetilde{\widetilde{V}}_{\varepsilon} / \rho(\mathrm{x})<\rho_{1}\right\}
\end{aligned}
$$

On the boundary of $\tilde{\tilde{V}}_{\varepsilon}$, either $-\widetilde{\widetilde{K}}_{\varepsilon}^{\prime}$ points inward $\widetilde{\tilde{V}}_{\varepsilon}$, or $\widetilde{\widetilde{K}}_{\varepsilon}$ is less than $c_{\varepsilon}-c_{2} \varepsilon$. Moreover, as $\widetilde{\widetilde{K}}_{\varepsilon}$ has no critical value between $c_{\varepsilon}-c_{2} \varepsilon$ and $c_{\varepsilon}-c_{1} \varepsilon, \widetilde{\widetilde{K}}_{\varepsilon}^{c_{\varepsilon}-c_{1} \varepsilon} \cap \widetilde{\tilde{V}}_{\varepsilon}$ retracts by deformation onto $\widetilde{\widetilde{K}}_{\varepsilon}^{c_{\varepsilon}-c_{2} \varepsilon} \cap \widetilde{\widetilde{V}}_{\varepsilon}$.

On the other hand, $-\rho^{\prime}$ points inward $\Omega_{d_{0}}^{k}$ on the boundary of this set for $d_{0}$ small enough and $\rho_{2}<\rho(\mathrm{x})<\rho_{1}$ (see Appendix B). As $\rho$ has no critical value between $\rho_{1}$ and $\rho_{2}$, we obtain that $\left\{(\lambda, \mathrm{x}) \in \tilde{\widetilde{V}}_{\varepsilon} / \rho(\mathrm{x})<\rho_{1}\right\}$ retracts by deformation onto $\left\{(\lambda, \mathbf{x}) \in \tilde{\widetilde{V}}_{\varepsilon} / \rho(\mathbf{x})<\rho_{2}\right\}$. Therefore

$$
\begin{aligned}
\left(\tilde{\tilde{V}}_{\varepsilon}, \widetilde{\widetilde{K}}_{\varepsilon}^{c_{\varepsilon}-c_{1} \varepsilon} \cap \tilde{\widetilde{V}}_{\varepsilon}\right) & \simeq\left(\tilde{\tilde{V}}_{\varepsilon},\left\{(\lambda, \mathrm{x}) \in \tilde{\widetilde{V}}_{\varepsilon} / \rho(\mathrm{x})<\rho_{2}\right\}\right) \\
& \simeq\left(\left\{\mathrm{x} \in \Omega_{d_{0}}^{k} / \rho(\mathrm{x})>\rho_{3}\right\},\left\{\mathrm{x} \in \Omega_{d_{0}}^{k} / \rho_{3}<\rho(\mathrm{x})<\rho_{2}\right\}\right) \\
& \simeq\left(\Omega_{d_{0}}^{k},\left\{\mathrm{x} \in \Omega_{d_{0}}^{k} / \rho(\mathrm{x})<\rho_{2}\right\}\right) \quad \text { by excision } \\
& \simeq\left(\Omega^{k},\left\{\mathrm{x} \in \Omega_{d_{0}}^{k} / \rho(\mathrm{x}) \leq 0\right\}\right) \\
& \simeq\left(\Omega^{k},\left\{\mathrm{x} \in \Omega^{k} / \rho(\mathrm{x}) \leq 0\right\}\right)
\end{aligned}
$$

since $-\rho^{\prime}$ point inward $\Omega^{k}$ for $d(\mathrm{x}, \partial \Omega)<d_{0}$ and $0<\rho(\mathrm{x})<\rho_{2}$, and $\rho$ has no critical value between 0 and $\rho_{2}$. This completes the proof of Theorem 2 .

\section{Appendix}

A - In this appendix, we prove that the least eigenvalue $\rho(\mathbf{x})$ of $M(\mathbf{x})$ is simple, and that the corresponding eigenvector may be chosen with all its components strictly positive. Indeed, we have

$$
\rho(\mathrm{x})=\inf _{r \in \mathrm{R}^{k}-\{0\}} \frac{r \cdot M(\mathrm{x}) \cdot{ }^{t} r}{|r|^{2}}
$$

and the corresponding eigenvectors are those elements of $\mathbf{R}^{k}$ for which the infimum is reached. 
Let $r=\left(r_{1}, \cdots, r_{k}\right)$ be such a vector. Then, $\bar{r}=\left(\left|r_{1}\right|, \cdots,\left|r_{k}\right|\right)$ is also an eigenvector for $\rho(\mathbf{x})$, since $|r|=|\bar{r}|$ and

$$
\begin{aligned}
\bar{r} \cdot M(\mathbf{x}) \cdot{ }^{t} \bar{r} & =\sum_{i=1}^{k} H\left(x_{i}, x_{i}\right) r_{i}^{2}-\sum_{\substack{1 \leq i, j \leq k \\
i \neq j}} G\left(x_{i}, x_{j}\right)\left|r_{i} r_{j}\right| \\
& \leq \sum_{i=1}^{k} H\left(x_{i}, x_{i}\right) r_{i}^{2}-\sum_{\substack{1 \leq i, j \leq k \\
i \neq j}} G\left(x_{i}, x_{j}\right) r_{i} r_{j} \\
& \leq r \cdot M(\mathbf{x}) \cdot{ }^{t} r
\end{aligned}
$$

Moreover, if $r_{i}=0$

$$
\begin{aligned}
\frac{\left(\bar{r}+\eta e_{i}\right) \cdot M(\mathrm{x}) \cdot^{t}\left(\bar{r}+\eta e_{i}\right)}{\left|\bar{r}+\eta e_{i}\right|^{2}} & =\frac{r \cdot M(\mathrm{x}) \cdot{ }^{t} \bar{r}-2 \eta \sum_{\substack{i, j \\
i \neq j}} G\left(x_{i}, x_{j}\right) \bar{r}_{j}+0\left(\eta^{2}\right)}{|\bar{r}|^{2}+\eta^{2}} \\
& <\frac{\bar{r} \cdot M(\mathbf{x}) \cdot{ }^{t} \bar{r}}{|\bar{r}|^{2}}=\rho(\mathrm{x})
\end{aligned}
$$

for $\eta>0$ small enough, in contradiction with the definition of $\rho(\mathbf{x})$. Thus there exists for $\rho(\mathbf{x})$ an eigenvector $r(\mathbf{x})$ whose all components are strictly positive. If the norm of $r(\mathbf{x})$ is imposed to be 1 , such a vector is unique, since $\rho(\mathbf{x})$ is simple. Indeed, let $\left(r(\mathbf{x}), r^{(1)}, \cdots, r^{(k-1)}\right)$ be an orthonormal basis of $\mathbf{R}^{k}$ composed with eigenvectors of $M(\mathrm{x})$. From $r(\mathrm{x}) \cdot{ }^{t} r^{(i)}=0, \forall i$, we infer that for each $i, r^{(i)}$ has some components which are striclty positive, some others which are strictly negative. Setting

$$
\bar{r}^{(i)}=\left(\left|r_{1}^{(i)}\right|, \cdots,\left|r_{k}^{(i)}\right|\right)
$$

we have $\left|r^{(i)}\right|=1$, and

$$
\rho(\mathbf{x}) \leq \bar{r}^{(i)} \cdot M(\mathbf{x}) \cdot{ }^{t} \bar{r}^{(i)}<r^{(i)} \cdot M(\mathbf{x}) \cdot{ }^{t} r^{(i)}=\rho^{(i)}
$$

This proves that $\rho(\mathrm{x})$ is simple

B - In this appendix, we are interested in the behavior of $\rho$ and $\tilde{F}$, for $\rho$ positive, near the boundary of $\Omega^{k}$. Namely, we want to prove that for $\mathbf{x}$ close enough to the boundary of $\Omega^{k}, \rho(\mathbf{x})>0$, then $-\rho^{\prime}$ and $-F^{\prime}$ are pointing inward $\Omega^{k}$. We may assume, without loss of generality, that $d_{1}=d\left(x_{1}, \partial \Omega\right)=\inf _{1 \leq i \leq k} d_{i}=$ $\inf _{1 \leq i \leq k} d\left(x_{i}, \partial \Omega\right)$. Thus, denoting by $u$ the outward normal to $\partial \Omega_{d_{1}}$ at $x_{1}$, we have to establish that

$$
\begin{aligned}
& \frac{\partial \rho}{\partial n}(\mathrm{x})=\frac{\partial \rho}{\partial x_{1}}(\mathrm{x}) \cdot n>0 \\
& \frac{\partial \tilde{F}}{\partial n}(\mathrm{x})=\frac{\partial \tilde{F}}{\partial x_{1}}(\mathrm{x}) \cdot n>0
\end{aligned}
$$

for any $\mathrm{x}$ such that $\rho(\mathrm{x})>0$ and $d_{1}$ is small enough. We have

$$
\tilde{F}^{\prime}(\mathrm{x})=\Lambda(\mathrm{x}) \cdot M^{\prime}(\mathrm{x}) \cdot{ }^{t} \Lambda(\mathrm{x})
$$


and

$$
\rho(\mathrm{x})=r(\mathrm{x}) \cdot M(\mathrm{x}) \cdot{ }^{t} r(\mathrm{x})=\inf _{|r|=1} r \cdot M(\mathrm{x}) \cdot{ }^{t} r
$$

Then, in order to prove (B.1) and (B.2), it is sufficient to prove that

$$
r(\mathrm{x}) \cdot \frac{\partial M}{\partial n}(\mathrm{x}) \cdot{ }^{t} r(\mathrm{x})>0
$$

and

$$
\Lambda(\mathrm{x}) \cdot \frac{\partial M}{\partial n}(\mathrm{x}) \cdot{ }^{t} \Lambda(\mathrm{x})>0
$$

As all the components of $r(\mathrm{x})$ and $\Lambda(\mathrm{x})$ are strictly positive, it is in fact sufficient to prove that all the components of $\frac{\partial M}{\partial n}(\mathrm{x})$ are positive, and $\frac{\partial M}{\partial n}(\mathrm{x}) \neq 0$. The components $m_{i j}$ of $\frac{\partial M}{\partial n}(\mathrm{x})$ are given by

$$
\left\{\begin{array}{l}
m_{i i}=\frac{\partial}{\partial n} H\left(x_{i}, x_{i}\right) \\
m_{i j}=-\frac{\partial}{\partial n} G\left(x_{i}, x_{j}\right) \text { if } \quad i \neq j
\end{array}\right.
$$

Of course, we have

$$
m_{i i}=0 \quad \text { if } \quad i \neq 1 ; m_{i j}=0 \quad \text { if } i \neq 1 \text { and } j \neq 1
$$

According to [26], we have the estimates

$$
\text { (B.5) } \frac{\partial H}{\partial a}(x, y)=-\frac{N-2}{\left|x-y+2 d_{x} n_{x}\right|^{N}}\left(x-y-2 n_{x} \cdot(x-y) n_{x}-2 d_{x} n_{x}\right)+\circ\left(\frac{1}{d_{x}^{N-1}}\right)
$$

as $d_{x}=d(x, \partial \Omega)$ goes to zero, $n_{x}$ denoting the outward normal to $\partial \Omega$ at $x^{\prime}$, where $x^{\prime}$ is the only point of $\partial \Omega$ such that $d_{x}=\left|x-x^{\prime}\right|$.

In particular

$$
\frac{\partial H}{\partial a}(x, x)=\frac{N-2}{2^{N-2} d_{x}^{N-1}} n_{x}+\circ\left(\frac{1}{d_{x}^{N-1}}\right)
$$

(B.6) implies that

$$
m_{i i}=\frac{\partial}{\partial n} H\left(x_{1}, x_{1}\right)=\frac{N-2}{2^{N-2} d_{1}^{N-1}}+\circ\left(\frac{1}{d_{1}^{N-1}}\right)
$$

since $n_{x_{1}}=n+o(1)$ and $\frac{\partial}{\partial x_{1}} H\left(x_{1}, x_{1}\right)=2 \frac{\partial H}{\partial a}\left(x_{1}, x_{1}\right)$.

Then, it is sufficient to prove that

$$
\frac{\partial}{\partial n} G\left(x_{1}, x_{j}\right) \leq 0 \quad j \neq 1
$$

We argue by contradiction. We assume that there exist a sequence $\left(\mathrm{x}^{n}\right) \in \Omega^{k}$ such that $\rho\left(\mathrm{x}^{n}\right)>0, d_{1}^{n} \rightarrow 0, d_{j}^{n} \geq d_{1}^{n}$, and $\frac{\partial}{\partial n} G\left(x_{i}^{n}, x_{j}^{n}\right)>0$. In the case where $\lim x_{1}^{n}=x_{1} \neq x_{j}=\lim x_{j}^{n}$, the strong maximum principle yields a contradiction. 
Suppose now that $x_{i}=x_{j}$. Two cases may occur :
1) $\frac{d_{j}^{n}}{d_{1}^{n}} \rightarrow+\infty$
2) $\frac{d_{j}^{n}}{d_{1}^{n}}$ is bounded

Let us consider the first case. We have

$$
\frac{\partial}{\partial n} G\left(x_{i}^{n}, x_{j}^{n}\right)=-(N-2) \frac{\left(x_{1}^{n}-x_{j}^{n}\right) \cdot n}{\left|x_{1}^{n}-x_{j}^{n}\right|^{N}}-\frac{\partial}{\partial n} H\left(x_{1}^{n}, x_{j}^{n}\right)
$$

and

$$
-(N-2) \frac{\left(x_{1}^{n}-x_{j}^{n}\right) \cdot n}{\left|x_{1}^{n}-x_{j}^{n}\right|^{N}} \sim-\frac{N-2}{\left(d_{j}^{n}\right)^{N-1}}
$$

On the other hand, as $y \mapsto \frac{\partial}{\partial x} H(x, y)$ is harmonic in $\Omega$ and is equal to $-(N-2) \frac{x-y}{|x-y|^{N}}$ on $\partial \Omega$, we have

$$
\frac{\partial}{\partial n} H\left(x_{1}^{n}, x_{j}^{n}\right)=(N-2) \int_{\partial \Omega} \frac{\partial G}{\partial \nu}\left(t, x_{j}^{n}\right) \frac{\left(x_{1}^{n}-t\right) \cdot n}{\left|x_{1}^{n}-t\right|^{N}} d t
$$

where $\frac{\partial}{\partial \nu}$ denotes the derivative with respect to the outward normal to $\partial \Omega$ at $t$. From the strong maximum principle $\frac{\partial G}{\partial \nu}<0$. As $\frac{\left(x_{1}^{n}-t\right) \cdot n}{\left|x_{1}^{n}-t\right|^{N}} \leq 0$ for $t \in \partial \Omega$ in a neighborhood of $x_{1}$, and $\frac{\partial G}{\partial \nu}\left(t, x_{j}^{n}\right) \frac{\left(x_{1}^{n}-t\right) \cdot n}{\left|x_{1}^{n}-t\right|^{N}}$ is bounded for $t \in \partial \Omega$ outside of this neighborhood, we obtain

$$
\frac{\partial}{\partial n} H\left(x_{1}^{n}, x_{j}^{n}\right) \geq 0(1)
$$

Finally

$$
\frac{\partial}{\partial n} G\left(x_{1}^{n}, x_{j}^{n}\right)=-\frac{N-2}{\left(d_{j}^{n}\right)^{N-1}}+\circ\left(\frac{1}{\left(d_{j}^{n}\right)^{N-1}}\right)<0
$$

for $n$ large enough, a contradiction with the assumptions.

Lastly, we deal with the second case. Note that $\rho\left(\mathbf{x}^{n}\right)>0$ implies that $\frac{d_{1}^{n}}{\left|x_{1}^{n}-x_{j}^{n}\right|}$ remains bounded. According to (B.5), we get

$$
\begin{aligned}
& \frac{\partial}{\partial n} G\left(x_{1}^{n}, x_{j}^{n}\right) \\
& =-(N-2) \frac{\left(x_{1}^{n}-x_{j}^{n}\right) \cdot n}{\left|x_{1}^{n}-x_{j}^{n}\right|^{N}}+\frac{N-2}{\left|x_{1}^{n}-x_{j}^{n}+2 d_{1} n\right|^{N}}\left(x_{1}^{n}-x_{j}^{n}-2 n \cdot\left(x_{1}^{n}-x_{j}^{n}\right) \cdot n-2 d_{1} n\right) n+\circ\left(\frac{1}{\left(d_{1}^{n}\right)^{N-1}}\right) \\
& \left(n_{x_{1}}=n+\circ(1)\right) \text {. From the equalities } \\
& \begin{aligned}
\left(x_{1}^{n}-x_{j}^{n}\right) \cdot n & =d_{j}^{n}-d_{1}^{n}+\circ\left(d_{1}^{n}\right) \\
\left|x_{1}^{n}-x_{j}^{n}+2 d_{1} n\right|^{2} & =\left|x_{1}^{n}-x_{j}^{n}\right|^{2}+4\left(d_{1}^{n}\right)^{2}+4 d_{1}^{n}\left(d_{j}^{n}-d_{1}^{n}\right)+\circ\left(\left(d_{1}^{n}\right)^{2}\right) \\
& =\left|x_{1}^{n}-x_{j}^{n}\right|^{2}+4 d_{1}^{n} d_{j}^{n}+\circ\left(\left(d_{1}^{n}\right)^{2}\right)
\end{aligned}
\end{aligned}
$$

we deduce that 


$$
\begin{aligned}
\frac{\partial}{\partial n} G\left(x_{1}^{n}, x_{j}^{n}\right) & =-(N-2) \frac{d_{j}^{n}-d_{1}^{n}}{\left|x_{1}^{n}-x_{j}^{n}\right|^{N}}-\frac{N-2}{\left(\left|x_{1}^{n}-x_{j}^{n}\right|^{2}+4 d_{1}^{n} d_{j}^{n}\right)^{N / 2}}\left(d_{j}^{n}+d_{1}^{n}\right)+\circ\left(\frac{1}{\left(d_{1}^{n}\right)^{N-1}}\right) \\
& <0 \text { for } n \text { large enough }
\end{aligned}
$$

again a contradiction with the assumptions. Therefore (B.7) holds, for $d_{1}=\inf d_{i}$ small enough and $\rho(\mathrm{x})>0$.

\section{References}

[1] Th. Aubin, Problèmes isopérimétriques et espaces de Sobolev, J. Diff. Geom. 11 (1976), 573-598.

[2] Th. Aubin, Equations différentielles non linéaires et problèmes de Yamabe concernant la courbure scalaire, J. Math. Pures et Appl. 55 (1976), 269-293.

[3] A. BAhri, Pitman Research Notes in Math. Series 182, Longman (1989).

[4] A. BAHri, J.M. Coron, Vers une théorie des points critiques à l'infini, Séminaire EDP Ecole Polytechnique (1984-1985), exposé nº 8 .

[5] A. Bahri, J.M. Coron, Une théorie des points critiques à l'infini pour l'équation de Yamabe et le problème de Kazdan-Warner, C.R. Acad. Sc. Paris 300 (1985), 513-516.

[6] A. Bahri, J.M. Coron, Sur une équation elliptique non linéaire avec l'exposant critique de Sobolev, C.R. Aca.d. Sc. Paris 301 (1985), 345-348.

[7] A. BAHri, J.M. Coron, On a nonlinear elliptic equation involving the critical Sobolev exponent : the effect of the topology of the domain, Comm. Pure Appl. Math. 41 (1988), 255-294.

[8] H. Brézis, L. Nirenberg, Positive solutions of nonlinear elliptic equations involving critical Sobolev exponents, Comm. Pure Appl. Math. 36 (1983), 437-477.

[9] H. Brézis, L.A. Peletier, in Asymptotics for elliptic equations involving the critical growth, Partial differential equations and the calculus of variations, F. Colombani, L. Modica, S. Spagnolo eds, Birkhauser (1989).

[10] L. Caffarelli, B. Gidas, J. Spruck, Asymptotic symmetry and local behavior of semilinear elliptic equations with critical Sobolev growth, CPAM 42 (1989), 271-297.

[11] E.N. Dancer, A note on an equation with critical exponent, Bull. London Math. Soc. 20 (1988), 600-602. 
[12] W.Y. Ding, Positive solutions of $\Delta u+u^{(n+2) /(u-2)}=0$ on contractible domains, to appear ().

[13] B. Gidas, in Symmetry properties and isolated singularities of positive solutions of nonlinear elliptic equations, in Nonlinear PDE in Engineering, Sternberg ed., Dekker, New-York (1980).

[14] B. Gidas, W. Ni, L. NirenberG, in Symmetry of positive solutions of nonlinear elliptic equations in $\mathbf{R}^{n}$, in Mathematical Analysis and Applications, Academic Press, L. Nachbin ed. (1981), 370-401.

[15] L. Glangetas, Uniqueness of solutions of a nonlinear elliptic equation involving critical exponent, to appear ().

[16] Z.C. Han, Asymptotic approach to singular solutions for nonlinear elliptic equations involving critical Sobolev exponent, Ann. Inst. Poincaré, Anal. non linéaire 8 (1991), 159-174.

[17] J. Kazdan, Prescribing the curvature of a Riemannian manifold, in C.B.M.S. Reg. Conf., A.M.S. (1985).

[18] J. KaZdan, F. WARner, Remarks on some quasilinear elliptic equations, Comm. Pure Appl. Math. 28 (1975), 567-597.

[19] C. LI, PhD Thesis, Courant Institute (1989).

[20] E. LIEB, Sharp constants in the Hardy-Littlewood-Sobolev and related inequalities, Ann. Math. (1983), 349-374.

[21] P.L. Lions, The concentration compactness principle in the calculus of variations The locally compact case, Ann. Inst. Poincaré, Parts I and II, Anal. non linéaire 1 (1984), 109-145 and 223-284.

[22] P.L. Lions, The concentration compactness principle in the calculus of variations The limit case, Rev. Mat. Iberoamericana 1.1., 1.2 (1985), 145-201 and $45-121$.

[23] M. OBATA, The conjectures on conformal transformations of Riemannian manifolds, J. Diff. Geom. 6 (1971), 247-258.

[24] S. Pohozaev, Eigenfunctions of the equation $\Delta u+\lambda f(u)=0$, Soviet Math. Dokl. 6 (1965), 1408-1411.

[25] O. REY, The role of the Green's function in a nonlinear elliptic equation involving the critical Sobolev exponent, J. Funct. Anal. 89 (1990), 1-52.

[26] O. REY, A multiplicity result for a variational problem with lack of compactness, J. Nonlinear Anal. TMA 13 (1989), 1241-1249. 
[27] O. REY, Sur un problème variationnel non compact: l'effet de petits trous dans le domaine, C.R. Acad. Sc. Paris 308 (1989), 349-352.

[28] O. REY, Bifurcation from infinity in a non linear elliptic equation involving the limiting Sobolev exponent, Duke Math J. 60 (1990), 815-861.

[29] O. REY, Blow-up points of solutions to elliptic equations with limiting nonlinearity, Differential Equations (1991).

[30] R. Schoen, Conformal deformation of a Riemannian metric to constant scalar curvature, J. Diff. Geom. 20 (1984), 479-495.

[31] R. Schoen, Graduate course in topics of differential geometry, given at Stanford University and Courant Institute (1988-1989).

[32] M. Struwe, A global compactness result for elliptic boundary value problem involving limiting nonlinearities, Math. Z. 187 (1984), 511-517.

[33] G. Talenti, Best constants in Sobolev inequality, Annali di Mat. 10 (1976), $353-372$.

[34] H. YAmABE, On a deformation of Riemanian structures on compact manifolds, Osaka Math. J. 12 (1960), 21-37.

Abbas BAHRI (1)(2), Yanyan LI (2), Olivier REY (1)

(1) Ecole Polytechnique, Centre de Mathématiques 91128 Palaiseau cedex, France

(2) Rutgers University, Department of Mathematics

New-Brunswick, NJ 08903, USA 